\title{
Retrotransposons evolution and impact on IncRNA and protein coding genes in pigs
}

\author{
Cai Chen ${ }^{1}$, Wei Wang ${ }^{1}$, Xiaoyan Wang ${ }^{1}$, Dan Shen', Saisai Wang ${ }^{1}$, Yali Wang ${ }^{1}$, Bo Gao ${ }^{1}$, Klaus Wimmers², \\ Jiude $\mathrm{MaO}^{3}, \mathrm{Kui} \mathrm{Li}^{4^{*}}$ and Chengyi Song ${ }^{\text {* }^{*}}$
}

\begin{abstract}
Background: Retrotransposons are the major determinants of genome sizes and they have shaped both genes and genomes in mammalian organisms, but their overall activity, diversity, and evolution dynamics, particularly their impact on protein coding and IncRNA genes in pigs remain largely unknown.

Results: In the present study, we performed de novo detection of retrotransposons in pigs by using multiple pipelines, four distinct families of pig-specific L1 s classified into 51 distinct subfamilies and representing four evolution models and three expansion waves of pig-specific SINEs represented by three distinct families were identified. ERVs were classified into 18 families and found two most "modern" subfamilies in the pig genome. The transposition activity of pig L1 was verified by experiment, the sense and antisense promoter activities of young L1 5'UTRs and ERV LTRs and expression profiles of young retrotransposons in multiple tissues and cell lines were also validated. Furthermore, retrotransposons had an extensive impact on IncRNA and protein coding genes at both the genomic and transcriptomic levels. Most protein coding and IncRNA (> 80\%) genes contained retrotransposon insertions, and about half of protein coding genes (44.30\%) and one-fourth (24.13\%) of IncRNA genes contained the youngest retrotransposon insertions. Nearly half of protein coding genes $(43.78 \%)$ could generate chimeric transcripts with retrotransposons. Significant distribution bias of retrotransposon composition, location, and orientation in IncRNA and protein coding genes, and their transcripts, were observed.
\end{abstract}

Conclusions: In the current study, we characterized the classification and evolution profile of retrotransposons in pigs, experimentally proved the transposition activity of the young pig L1 subfamily, characterized the sense and antisense expression profiles and promoter activities of young retrotransposons, and investigated their impact on IncRNA and protein coding genes by defining the mobilome landscapes at the genomic and transcriptomic levels. These findings help provide a better understanding of retrotransposon evolution in mammal and their impact on the genome and transcriptome.

Keywords: Pig genome, Retrotransposon evolution, Gene overlapping, Retrotransposition activity, Promoter activity, Distribution bias

\section{Background}

Transposable elements (TEs), also referred to as the mobilome, are DNA sequences that have the ability to integrate into the genome at a new site within their cell of origin. They can be divided into retrotransposons and DNA transposons based on their diverse structures and

\footnotetext{
*Correspondence: likui@caas.cn; cysong@yzu.edu.cn

${ }^{4}$ Institute of Animal Science, Chinese Academy of Agricultural Sciences, Beijing, China

'Institute of Animal Mobilome and Genome, College of Animal Science \&

Technology, Yangzhou University, Yangzhou 225009, Jiangsu, China

Full list of author information is available at the end of the article
}

transposition mechanisms. Retrotransposons consists of short interspersed elements (SINEs), long interspersed elements (LINEs), and long terminal repeats (LTRs), including endogenous retroviruses (ERVs), all of which propagate by the reverse transcription (RT) of an RNA intermediate $[1,2]$. TEs were once viewed merely as junk DNA and selfish DNA parasites. However, genomescale studies over the past several decades have shown that TEs and their recognizable remnants span both prokaryote and eukaryote organisms, are major determinants of genome sizes [3-5], and account for about half of the human genome [6]; they even make up $85 \%$ of the

(c) The Author(s). 2019 Open Access This article is distributed under the terms of the Creative Commons Attribution 4.0 International License (http://creativecommons.org/licenses/by/4.0/), which permits unrestricted use, distribution, and 
maize genome [7]. TEs have shaped both genes and the entire genome and play a key role in genome function, speciation, and diversity $[8,9]$. TEs also contribute substantially to the evolution of the genome at the DNA level, and they can undergo "molecular domestication" [10, 11]; at least 50 genes have been domesticated from mobile elements in the human genome [12]. Chimeric transcripts between TEs and protein coding genes tend to be common $[13,14]$. TE insertions can also induce diverse structural variations of the genome $[9,15]$. Furthermore, TEs contribute substantially to the evolution of many genes at the transcriptional level by acting as alternative promoters, enhancers, splice sites, or polyadenylation signals [16, 17], or the transcription factor binding sites for these genes [18]. It has also been suggested that a majority of primate-specific regulatory sequences are derived from TEs [19]. The epigenetic landscape can be altered by TE insertions [20]. Evolution of the sperm methylome of primates is associated with Alu and SVA retrotransposon insertions [21]. Methylation levels of retrotransposons are associated with carcinogenesis and metastasis [22-24]. In addition, growing evidence shows a close association of TEs with noncoding RNAs (ncRNAs), and a significant number of small ncRNAs originate from TEs [25]. Furthermore, TEs tend to enrich in the lncRNAs of human, mice, and zebrafish $[26,27]$, and retrotransposons make a strong contribution to IncRNA evolution, structure, and function in mammalian organisms [28].

Retrotransposons occupy one-third to half of the mammal genomes, which are dominated by LINEs and SINEs, followed by LTR retrotransposons [8]. The LINE family is the most successful TE family in both the common ancestors and extant species of mammals, and account for $20.42 \%$ in humans, $19.20 \%$ in mice, $19.54 \%$ in pigs, $21.21 \%$ in platypuses, and $28.60 \%$ in tammars, while SINEs, known as the partner of LINEs that require LINEs for their transposition, account for 13.14\% in humans, $8.22 \%$ in mice, $13.08 \%$ in pigs, $21.53 \%$ in platypuses, and $11.70 \%$ in tammars. LTRs are the third major type of interspersed repeats in mammals, accounting for $8.29 \%$ in humans, $9.87 \%$ in mice, $4.48 \%$ in pigs, $0.12 \%$ in platypuses, and $3.90 \%$ in tammars $[6$, 29-32]. In addition, unusual evolution dynamics of L1 s in mammals are observed, with a single family of replicative dominant subfamilies evolved in one period, then being replaced by a more recently evolved family [33]. Studies in humans and mice also revealed the diversification evolution of $\mathrm{L} 1 \mathrm{~s}$, and the coexistence of multiple L1 subfamilies with different promoters in young and ancient families [34-36]. Little is known about the factors that determine the burst and decline of SINEs, but, clearly, SINE amplification is dependent on LINE activity, and activity correlation is observed for many SINE/LINE partners; for example, mammalian-wide interspersed repeat (MIR) (Ther-1) and L2 in humans and mice [6, 29], MEG and L1 in fruit bats [37, 38], and Alu and L1 subfamilies in humans [39]. Although most retrotransposons are no longer active in mammals, research has shown that most mammal genomes contain at least one family of actively accumulating retrotransposons [8, 40]. Examples include L1/LINEs in most mammals [41], RTE/LINEs in ruminants and marsupials [42], with the exception of LINEs in the megabat family, where the activity of L1 went extinct 24 million years ago [43], while ERVs/LTR in rodent genomes are believed to be active $[29,44]$. The retrotransposition activities of L1 s and SINEs in humans and mice, including human $\mathrm{L} 1\left(\mathrm{~L} 1 \mathrm{H}_{\mathrm{S}}\right)$, mouse $\mathrm{L} 1\left(\mathrm{~T}_{\mathrm{F}}\right.$ and $\left.\mathrm{G}_{\mathrm{F}}\right)$, and both human SINE (AluYa5/8 and AluYb8/9) and mouse SINE (SINE B1 and SINE B2), have been verified experimentally [41].

Despite the prevalence of retrotransposons in mammalian genomes and their biological relevance, relatively few pig retrotransposons have been reported. Initially, the TE coverages in Duroc and Wuzhishan pig genomes have been well annotated in previous studies $[30,45]$. The divergence distribution and phylogenetic analysis of retrotransposons in pigs revealed that the main repeating element groups are LINEs and SINEs, and only a single family of each is deduced to be putatively active [30,45], and two complete pig ERVs were identified in Wuzhishan pig genome, which may carry the risk of pathogen transmission to human in xenotransplantation [45]. Whereas the overall activity, diversity, and evolution of retrotransposons, particularly the diversity at the family, and subfamily levels, and the evolution dynamics of the dominate L1, SINE, and ERV families, in the pig genome remain largely unknown. In addition, retrotransposon involvement in the structural and functional evolution of genes and genomes, as well as their impact on the transcriptome in pig, remain completely unknown.

In this study, we performed de novo detection of retrotransposons in pigs using multiple pipelines. We characterized the classification of LINEs, SINEs, and ERVs at the family and subfamily levels, highlighted the evolution dynamics of these families and subfamilies, and then determined the retrotransposition activity of L1 and the sense and antisense promoter activities and expression profiles of young retrotransposon subfamilies. Furthermore, we investigated the intersection between retrotransposons and host genes, including protein-encoding and lncRNA genes, as well as the impact of retrotransposons on the transcriptome. Overall, this study revealed the retrotransposon landscape and their evolution profiles in the pig genome, domesticated the retrotransposition activities of young L1 subfamilies, and defined the sense and antisense expression 
profiles and promoter activities of young retrotransposon subfamilies. Our data support the hypothesis that most copies of retrotransposons are fossils in the pig genome, but a few retrotransposon copies of L1 s, SINEs, and ERVs may still be active. Our analysis also reveals that the majority of protein coding and lncRNA genes contain retrotransposon insertions, and retrotransposons tend to be enriched in lncRNA, with nearly half of protein coding genes generating chimeric transcripts with retrotransposons.

\section{Results}

Four distinct families of pig-specific L1 s representing four evolution models

A total of 4154 L1 elements were identified by MGEScan-non-LTR, and they were aligned against the pig genome by Blat with an extension of $2500 \mathrm{bp}$ of $5^{\prime}$ UTR and $200 \mathrm{bp}$ of 3 'UTR to get the full lengths of the elements. In addition, $4495 \mathrm{~L} 1$ elements were downloaded from L1Base database and merged with these L1 s, and the redundancy was removed. Finally, we obtained $5937 \mathrm{~L} 1$ elements with unique position in the pig genome. These L1 s were classified into 51 distinct subfamilies, including one subfamily (L1_B-SS) deposited in Repbase, according to their 5'UTR sequences, and consensus sequences were derived for each. Two subfamilies (HAL1_Ssc and L1_3_Ssc) deposited in Repbase, but not detected by our protocol, were also included for annotation. A few older subfamilies with too few $(<10)$ copy numbers to derive accurate consensus sequences were removed from the dataset. It is very likely that additional, ancient, small copy number subfamilies exist, but were missed by our approach. The remaining subfamilies were further classified into four distinct families (named L1A, L1B, L1C, and L1D) based on the polygenic tree of $5^{\prime}$ UTR (Fig. 1). The names, classification, characteristics, divergence, and copy numbers of these L1 $\mathrm{s}$ are summarized in Table 1 and Additional file 2: Table S1, and the consensus sequences of each subfamily are supplied in Additional file 1 . The total length of the consensus varied between 5837 and $8822 \mathrm{bp}$, while the length of the 5'UTR varied widely from $551 \mathrm{bp}$ to $3254 \mathrm{bp}$, and the 3 'UTR (excluding polyA sequence) varied from $180 \mathrm{bp}$ to $305 \mathrm{bp}$ between subfamilies. The intergenic region (IGR) ranged from $390 \mathrm{bp}$ to $529 \mathrm{bp}$, except two subfamilies (L1A1 and L1A2) containing very short IGRs (67 and $68 \mathrm{bp}$ ), while the lengths of open reading frame 1 (ORF1) (about $900 \mathrm{bp}$ ) and ORF2 (about $3800 \mathrm{bp}$ ) were relatively conservative across all subfamilies and families (Table 1 and Additional file 2: Table S1). The copy number of L1 elements, number of subfamilies, divergences, and the copy number of full length L1 elements varied significantly between families. The number of subfamilies across L1A, L1B, and L1C families, and the copy number of elements in each subfamily are generally similar, but subfamily L1A4 of L1A tended to show more elements compared with the other subfamilies of L1A, L1B, and L1C families. The family L1D represents the highest diversity, with 22 subfamilies, and this family also displays the highest activity, with several subfamilies containing members with the potential to encode, and most subfamilies show lower divergence compared with other families (Table 1 and Additional file 2: Table S1). In total, 98 putatively active L1 elements with a typical structure of mammal L1 were identified, and they distributed in 12 different subfamilies of L1D family. Most of them tend to have a longer 5 'UTR compared with other subfamilies (Table 1 and Additional file 2: Table S1). Thus, in the pig genome, the putatively active L1 elements are 7-9 $\mathrm{kb}$ long and contain a $5^{\prime} \mathrm{UTR}$ with length ranging from $1.5 \mathrm{~kb}$ to $3.2 \mathrm{~kb}$, a ca. $270 \mathrm{bp} \mathrm{3'UTR,} \mathrm{two} \mathrm{open-reading}$ frames (296 aa ORF1 and 1272 aa ORF2), and a relatively long (ca. $520 \mathrm{bp}$ ) IGR that separates the two ORFs. L1 insertions typically end with an A-rich tail and are flanked by short ( $<20$ bp) target site duplication (Fig. 2a).

Analysis of the age distribution between the pig-specific L1 families (L1A, L1B, L1C, L1D) other L1 families (mammal common), L2 superfamily, and other LINEs revealed that the mammal common $\mathrm{L} 1 \mathrm{~s}, \mathrm{~L} 2$, and other LINEs were fossils, represented ancient proliferation, and dominated the genome evolution between 30 and 80 million years ago (Mya); their activities have essentially ceased for over 30 million years (Fig. 2b), while the four pig-specific families of L1 have dominated evolution over the last 30 Mya. Further comparison of the age distribution across the four families clearly showed that they proliferated at different evolutionary periods and represented variable evolutionary profiles (Fig. 2b). Generally, both the L1A and L1B families displayed an extended accumulation during their evolutionary history; they amplified and evolved simultaneously for as long as 80 Mya, from 90 Mya to 10 Mya, and burst between 10 and 20 Mya. By contrast, both the L1C and L1D families amplified over the last 20 Mya in the evolution of the pig genome. L1C displayed a low expansion between 5 Mya and 20 Mya and tended to be dead in the last 5 Mya, whereas L1D represented the youngest and most active family in the pig L1 clade and showed a sharp amplification in the last $10 \mathrm{Mya}$, with peak activity at 5 Mya; indeed, they are potentially still active (Fig. 2b), which is also consistent with the results of age analysis and the identification of about 100 putatively active L1 elements in this family. Further analysis revealed that L1D1-7 subfamilies may represent the youngest subfamilies across this family, compared with other subfamilies (L1D8-21) (Fig. 2c and d), with each subfamily containing many putatively active L1 copies. This observation 


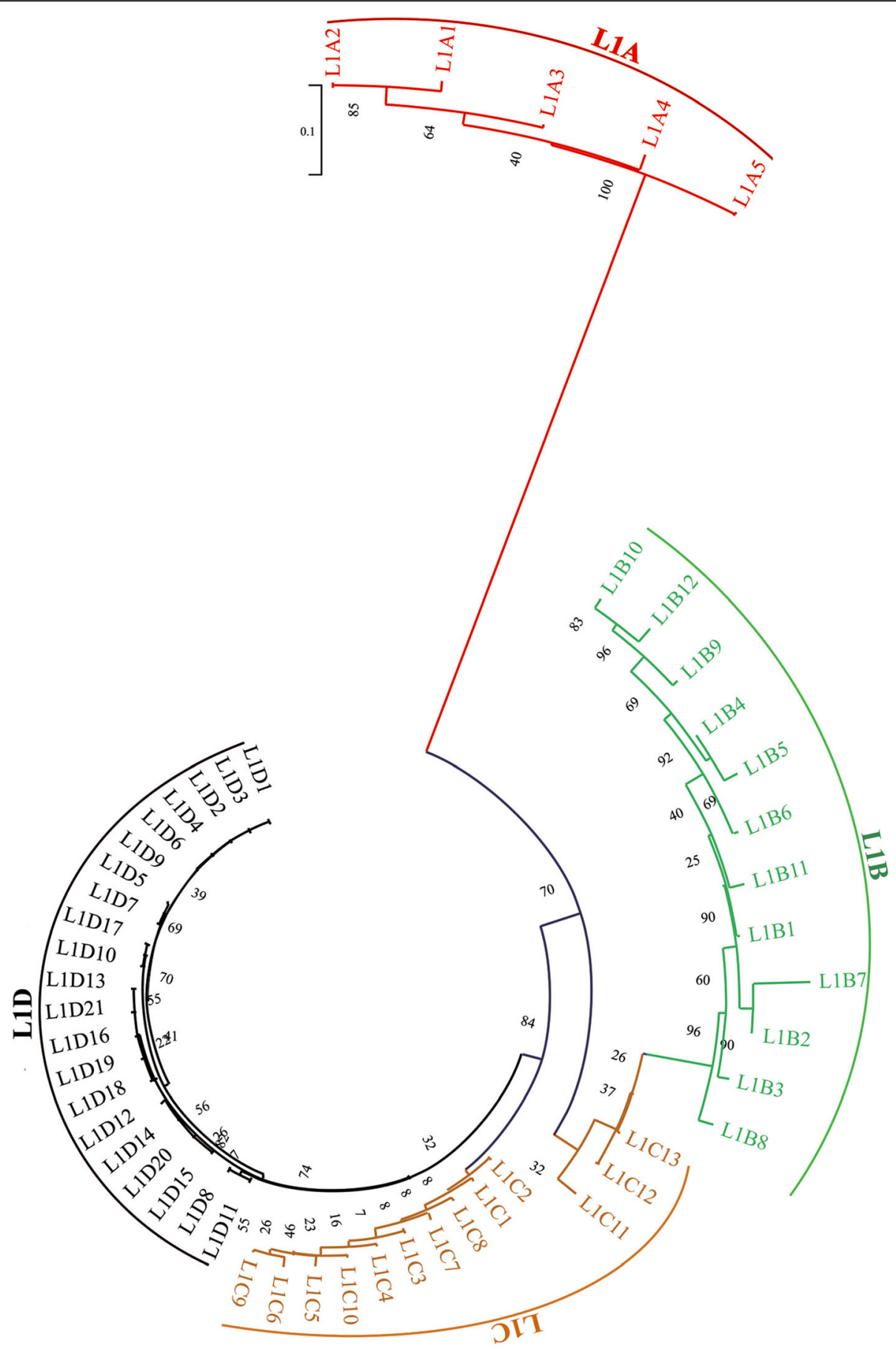

Fig. 1 Neighbor-joining polygenic tree of pig L1 based on the 5'UTR and classified L1 s into four distinct families (L1A, L1B, L1C, and L1D)

was also well supported by the insertion polymorphism analysis of L1D1 in both inter- and intrabreed pigs (Fig. 2e). The long history of expansions means that the abundance of most subfamilies of families L1A and L1B was significantly higher than that of L1C and L1D (Table 1 and Additional file 2: Table S1).
Three expansion waves of pig-specific SINEs represented by three distinct families

Diverse pig-specific SINE elements have been identified in a previous study [30] and deposited in Repbase (https://www.girinst.org/), and all these SINEs are tRNA-derived. We also tried to use MITE-Hunter, which 
Table 1 Classification of L1 families in the pig Genome

\begin{tabular}{|c|c|c|c|c|c|c|c|c|}
\hline \multirow{2}{*}{$\begin{array}{l}\text { L1 } \\
\text { family }\end{array}$} & \multirow{2}{*}{$\begin{array}{l}\text { Subfamily } \\
\text { Number }\end{array}$} & \multicolumn{6}{|l|}{ Length (bp) } & \multirow{2}{*}{$\begin{array}{l}\text { Active } \\
\text { L1 } \\
\text { Numbe }\end{array}$} \\
\hline & & Consensus & $5^{\prime} U T R$ & ORF1 & IGR & ORF2 & 3'UTR (No PolyA) & \\
\hline L1A & 7 & $5837-7404$ & 931-1959 & $897-906$ & $67-396$ & $3655-3828$ & 180-305 & \\
\hline L1B & 12 & $5975-7740$ & $551-2335$ & 878-910 & $390-447$ & $3766-3813$ & 217-305 & \\
\hline L1C & 13 & $6462-7532$ & $1037-2024$ & 879-891 & $385-529$ & $3766-3814$ & $247-268$ & \\
\hline L1D & 21 & $7072-8822$ & $1562-3254$ & 887-891 & $501-521$ & $3807-3819$ & $270-277$ & 98 \\
\hline
\end{tabular}

is a program for discovering miniature inverted-repeat TEs from genomic sequences and can be used to identify SINEs, and RepeatModeler to extract SINE elements; however, we did not find any new families. Thus, these SINEs in Repbase were classified into three families (named SINEA, SINEB, and SINEC) based on length and structure, as shown in Additional file 2: Figure S1 and Table S2 (family, new name and Repbase name, length) and Additional file 1. All SINE elements of SINEA, SINEB, and SINEC families showed similar structure organization, with a tRNA head, a TC-rich region, a GC-rich region, and an A-rich tail (Fig. 2a). Similar to Alu in humans [46] and B1 and B2 in mice [47], the tRNA head of pig SINE harbors the conserved A and $\mathrm{B}$ box sequences that are required for RNA polymerase III dependent transcription. The TC-rich region also presents in carnivore SINE elements [48]. The elements of SINEA family are approximately $250 \mathrm{bp}$ in length, with the exception of a polyA tail, while the elements of SINEB and SINEC family are shorter, with about $200 \mathrm{bp}$ and $120 \mathrm{bp}$ lengths, respectively. Sequence length variations between the $A$ and $B$ box sequences of the tRNA head of SINEB and SINEC families' elements have been observed, whereas the SINEA elements are highly conserved and display high sequence similarity, indicating that SINEA may represent the youngest family (Additional file 2: Figure S1 and Table S2).

Three waves of expansion of SINEs can be identified in pigs based on the tempo of their evolution, and each wave corresponds to the activity of one family. However, most of these families, including SINEB, SINEC, MIR, and others, have been extinct for at least 20 million years (Fig. 2f); the most recent expansion corresponded with the activity in the family of SINEA. This family dominated the evolution history of SINEs in the pig genome during the last $50 \mathrm{Mya}$, and still displayed activity during the last 10 Mya. Three subfamilies (SINEA1, SINEA2, and SINEA3) of this family represented the youngest SINE elements compared with other subfamilies (SINEA4-11), and may currently be active, with many copies inserted in the last 5 million years (Fig. $2 \mathrm{~g}$ and $\mathrm{h}$ ); this was also supported by the insertion polymorphisms of SINEA1 in both inter- and intrabreed pigs (Fig. 2e). While the MIR represents the oldest family, its retrotransposon activity peaked approximately 65 Mya, and SINEB and SINEC represents the second oldest family; its retrotransposon activity peaked approximately 40-45 Mya (Fig. 2f).

\section{Experimental evidence for the Retrotransposition competence of pig L1}

To determine the retrotransposition activities of L1, we used a retrotransposition assay with an indicator cassette consisting of blasticidin resistance gene in the antisense orientation (relative to L1) that is disrupted by an intron ( $\gamma$-globin 2) in the sense orientation, which becomes functional only after a cycle of transcription, removal of the intron by splicing, RT, and integration [49-51]. We cloned the 5'UTR, ORF1, IGR, ORF2, and 3'UTR from the genomic coordinate of the youngest L1 (L1D1) subfamily and inserted it into the retrotransposon activity verification vectors, respectively, as described in the methods. We also used CMV as promoter to replace the 5'UTR of pig L1, and IGR of human L1 to replace the pig IGR. Human active L1 vector, which contains the most active L1 copy from the human genome, and mutant $\mathrm{L} 1$ vector, which is the same as active $\mathrm{L} 1$ vector but has an ORF1 mutant and cannot support retrotransposition [50], were used as positive and negative controls, respectively. The schematics of the constructs used are listed in Fig. 3a. We found that the cloned pig L1 was capable of retrotransposition in HeLa cells either with pig 5'UTR or CMV, but in a low level of retrotransposition activity compared with human L1 (Fig. $3 \mathrm{~b}$ and c). Replacement of the pIGR with human IGR can improve the retrotransposition activity significantly. We also found that the retrotransposition activity of pig and human L1 s were cell-specific; weak retrotransposition activity of pig and human chimeric L1 (phL1) was observed in porcine kidney (PK15) cells, whereas human L1 did not work in the PK15 cell line (Fig. 3b and c).

\section{Identification of the Most "modern" ERV in the pig genome}

LTRharvest and RetroTector pipelines were used to detect ERVs in the pig genome DNA. A total of 2120 and 5456 ERV candidates were identified by using RetroTector and LTRharvest, respectively. Only ERVs 


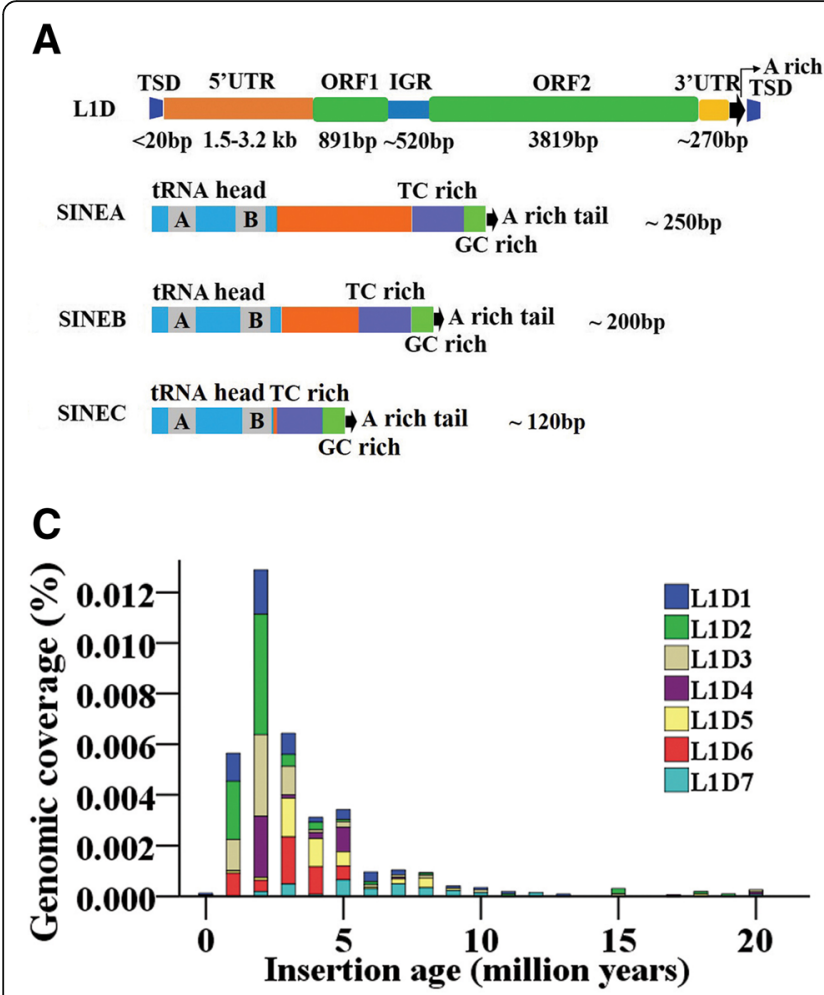

\section{B}

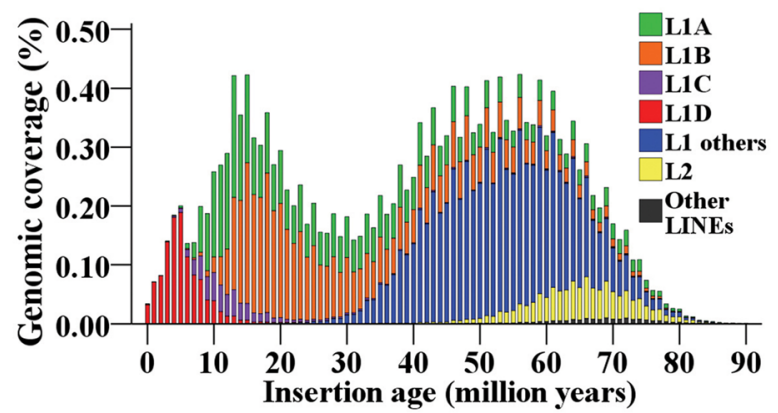

D

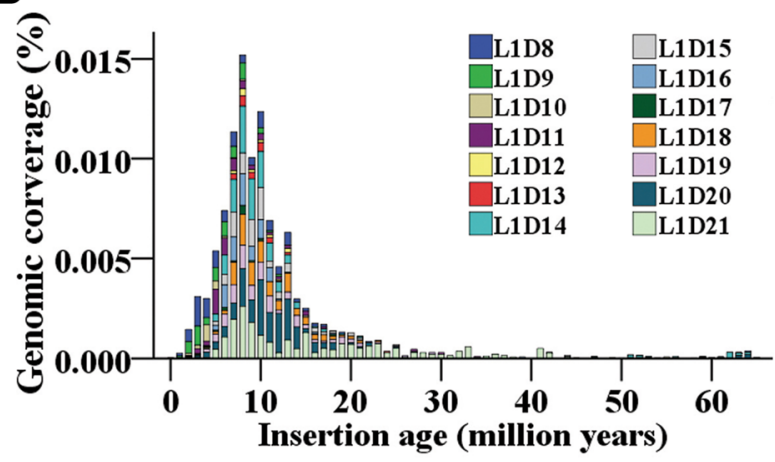

E

F
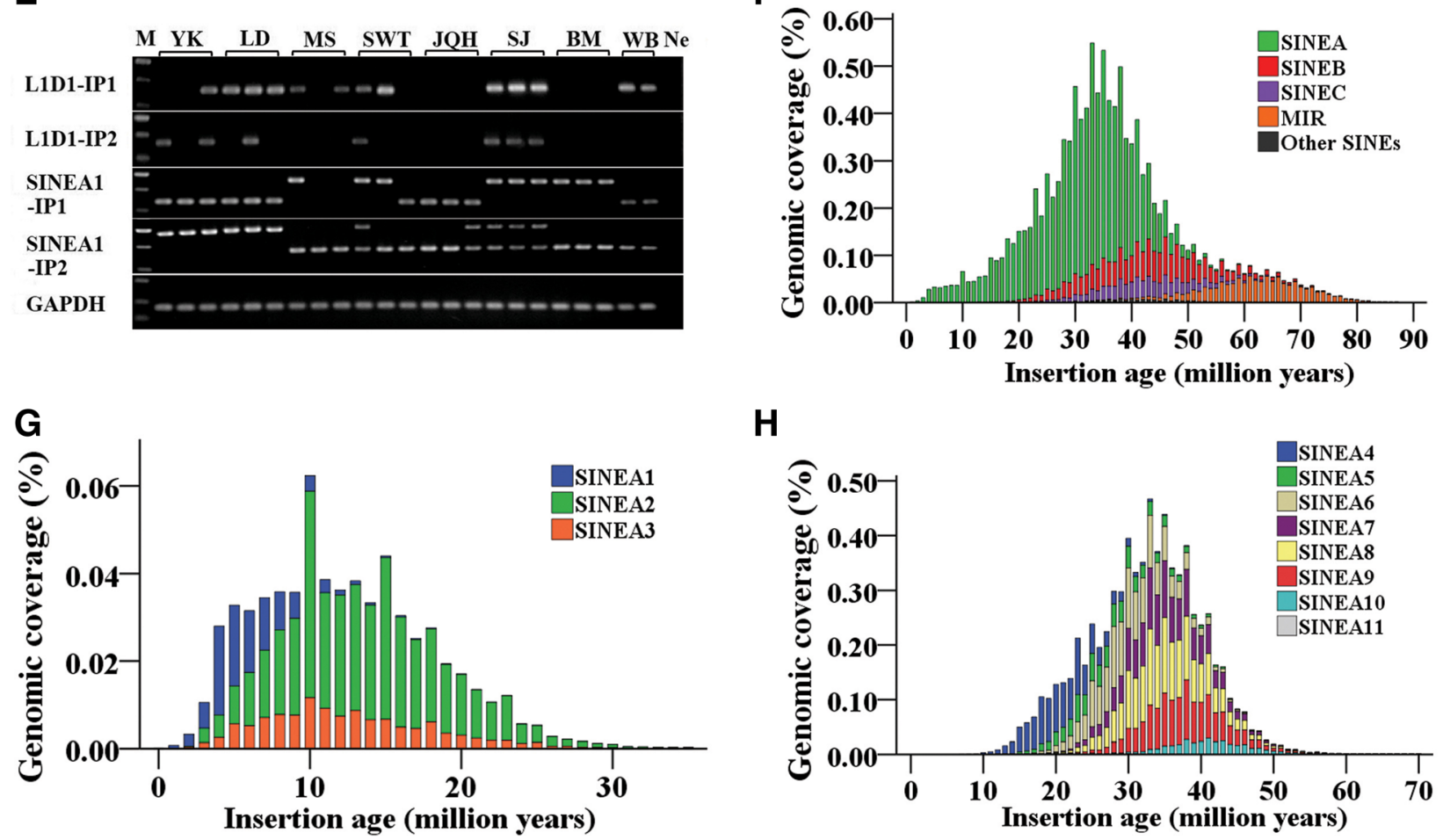

H

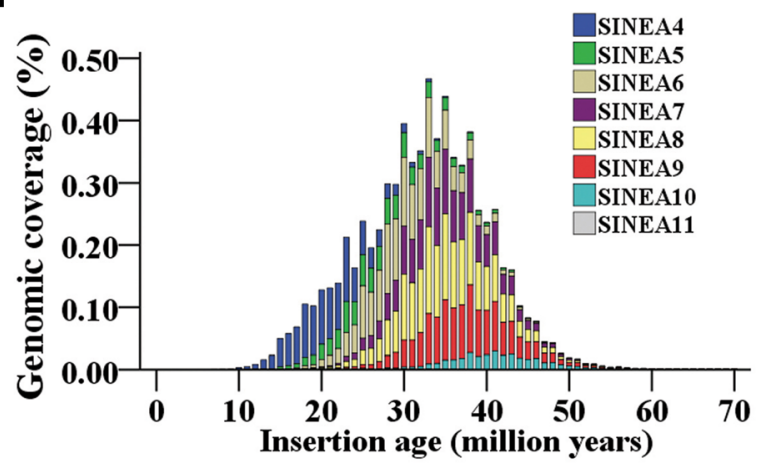

Fig. 2 (See legend on next page.) 
(See figure on previous page.)

Fig. 2 Evolution of L1 $s$ and SINEs in the pig genome. a Structural schematics of the putatively active L1 s and pig-specific SINE families (SINEA, SINEB, and SINEC). $\mathbf{b}$ Age distribution of pig-specific L1 families. $\mathbf{c}$ and $\mathbf{d}$ Age distribution across the subfamilies (L1D1-21) of the youngest L1 family (L1D). e Insertion polymorphism (IP) detection of the youngest L1 (L1D1) and SINE (SINEA1) subfamilies by PCR. Breed name abbreviations: Meishan (MS), Shawutou (SWT), and Jiangquhai $(\mathrm{JQH})$ pigs are native Chinese pig breeds from Jiangsu Province; the Sujiang (SJ) pig is a newly established breed based on the Duroc and Jiangquhai bloodlines; Bama (BM) pigs are miniature pigs from Guangxi Province; the wild boar (WB) was from Anhui Province; and the Landrace (LD) and Yorkshire (YK) pigs were from a breeding farm in Anhui Province. Ne, negative control without DNA. Two transposon loci in each of the youngest transposon subfamilies were selected for insertion polymorphism (IP) detection and labeled as IP1 and IP2. If an individual contains SINE insertion at SINEIP1 or SINE-IP2 site, the band size would be 629 or 676 bp, respectively, and if no SINE insertion, the band would be 335 or 382 bp. The three bands showed in the M (marker) lane are $750 \mathrm{bp}, 500 \mathrm{bp}$ and $250 \mathrm{bp}$ from top to bottom. f) Age distribution of pig-specific SINE families. $\mathbf{g}$ and $\mathbf{h}$ Age distribution across the subfamilies (SINEA1-11) of the youngest SINE family (SINEA). The $x$-axis represents the insertion age (Million years ago, Mya), and the $y$-axis represents the percentage of the genome composed of retrotransposon families/subfamilies (\%) in Fig. $\mathbf{b}, \mathbf{c}, \mathbf{f}$, and $\mathbf{g}$

with intact RT regions (ca. $0.7 \mathrm{~kb}$ ) were retained, resulting in 29 and 240 ERVs from LTRharvest and RetroTector, respectively. They were then used for the subsequent phylogenetic analysis (Table 2 and Additional file 2: Table S3). These ERVs were classified into 18 families (ERV1-ERV18), including six families deposited in Repbase, based on the phylogenetic tree: 13 as gamma retroviruses of class I (ERV113), three as beta retroviruses of class II (ERV15-18), and one as spuma of class III (ERV14) (Fig. 4a, Additional file 2: Figure S2, and Table S3). The number of ERVs containing RT regions varied greatly among the types of retroviruses and families. Gamma retroviruses tended to have more ERV families and elements than did beta and spuma retroviruses. ERV candidates featuring two LTRs and three structural polyproteins common to all retroviruses, including group-specific antigen $(g a g)$, polymerase $(p o l)$, and envelope protein (env), were designated as full ERVs (Fig. $4 \mathrm{~b}$ and Additional file 2: Figure S3). Most of the ERV families had decayed in pigs and tended to be inactive; only 19 copies of non-redundant ERV candidates were identified as full ERVs. Two of the latter, with the ability to encode long ERV proteins, were putatively active, and designated as "modern" ERVs (Fig. 4b and Additional file 2: Figure S3). All of the full and active ERVs identified in this study, as well as the transfection competent pig ERVs $(\gamma 1 \mathrm{~A}, \gamma 1 \mathrm{~B}$, and $\gamma 1 C$ ) identified in previous studies [52], were classified in the ERV6 family of gamma retroviruses, which were further classified into ERV6A and ERV6B subfamilies based on LTRs (Additional file 2: Table S3). The consensus or representative sequences were derived for each family or subfamily (Additional file 1; Additional file 2: Table S3). Most ERVs were typically between $8.5 \mathrm{~Kb}$ and $11 \mathrm{~Kb}$ in length, and the length of LTRs varied from 110 to $702 \mathrm{bp}$. Each of the two youngest subfamilies of ERVs (ERV6A and ERV6B) contained one putatively active ERV element with lengths of $8918 \mathrm{bp}$ (chr5:92185133-92,194,050 -) and 8757 bp (chr9:138895584-138,904,340 -), respectively. The putatively active ERV element of ERV6A encoded an 1, 748 aa peptides containing gag, pol, and env, which are essential for replication, and flanked with 702 bp LTRs, while the active ERV of ERV6B subfamily encoded an 1, 776 aa peptide harboring gag, pol, and env, but flanked with 629 bp LTRs (Fig. 4b and Additional file 2: Figure S3).

Overall, the expansion profile of the three classes of ERVs was very different in the pig genome. Class I and III ERVs displayed abundant amplification and dominated the whole evolution history of ERVs in the pig genome, whereas class II ERVs were the least abundant and showed a very weak expansion during the whole evolution history of ERVs. The other LTRs, including Gypsy, displayed extremely low amplification. Most of the ERV families appeared to be defective, with a striking deceleration in activity over the last 10 million years, and most of them seemed to cease in the most recent 5 million years (Fig. 4c). However, one possible exception was the family of ERV6, which exhibited an extended expansion between 30 and 0 Mya and a burst in the last 10 million years, and displayed signs of current activity. By contrast, the ERV6B subfamily may represent the youngest ERVs in the pig genome (Fig. 4d), combining the insertion polymorphisms detection of ERV6B by PCR in both inter- and intrabreed pigs (Fig. 4e), strongly suggesting that the current activity of this subfamily may represent the most "modern" ERV.

\section{Young L1 5'UTRs and ERV LTRs displayed sense and antisense promoter activities}

The sense and antisense LTRs from the putatively active family of ERV (ERV6A and ERV6B) were cloned into the pGL3 luciferase reporter vector to investigate the promoter activity based on the luciferase assay; the vector schematics are shown in Fig. 5a. ERV6B sense LTR had the highest promoter activity in three tested cell lines, while ERV6A sense LTR and ERV6B antisense LTR showed moderate promoter activity. The promoter activity of ERV6A antisense LTR was not detectable (Fig. 5b). We also explored the promoter activities of eight sense and four antisense 5'UTRs from young and putatively active subfamilies of L1D. Four of the sense 5'UTRs were members of the L1D1, L1D4, L1D6, and L1D7 subfamilies, and two of them were 


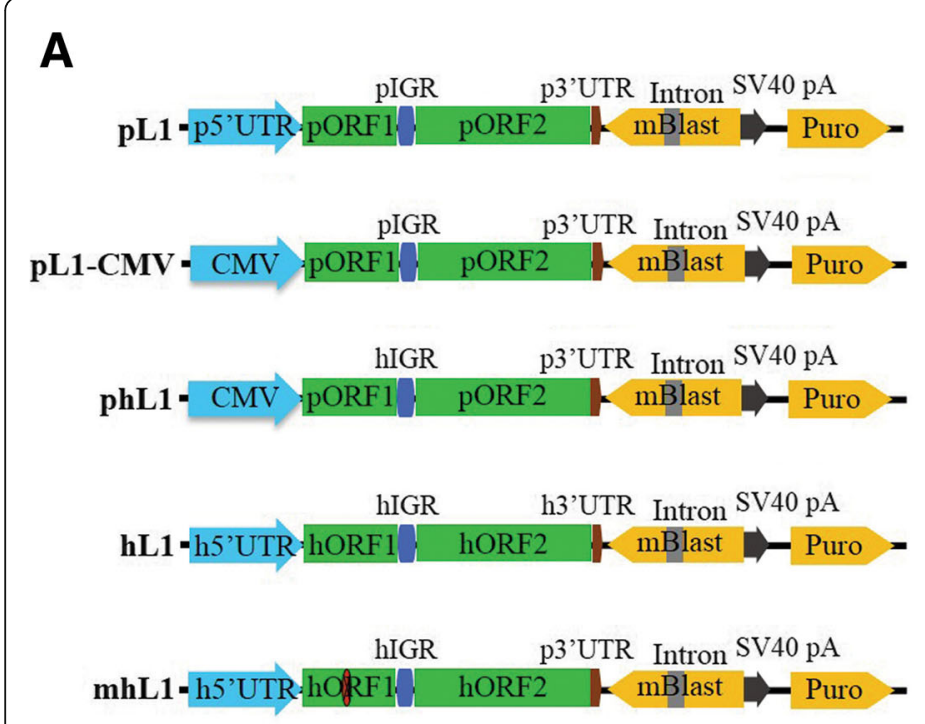
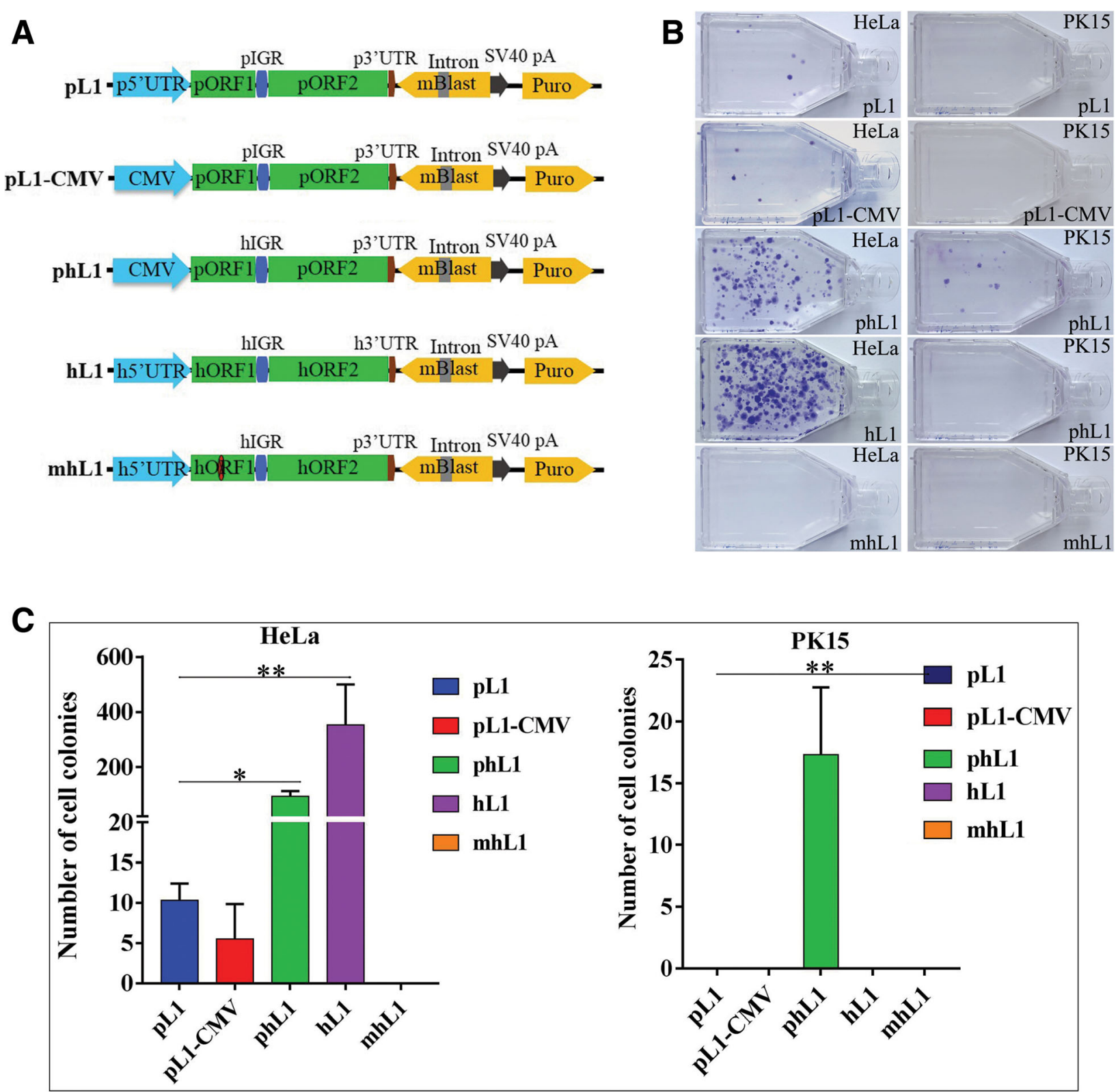

Fig. 3 Retrotransposition activity analysis of pig L1. a Schematics of vectors used for retrotransposition assays. hL1 and mhL1 were used as positive and negative control, respectively. The pL1 vector contains 5'UTR, ORF1, IGR, ORF2, and 3'UTR of L1 cloned from the pig genome (L1D1 coordinate). The pL1CMV is the same as $\mathrm{LL} 1$, but the $5^{\prime} \mathrm{UTR}$ of pig L1 was replaced with the CMV promoter. The phL1 is a chimeric vector derived by the CMV promoter, the two ORFs and 3'UTR were from pig, and the IGR was from human L1 (99-PUR-RPS-pBlaster1). All the vectors contain two selective cassettes (mBlast and Puro) for two-round selections. The mBlast cassette contains an inverted blasticidin resistance gene (black box) disrupted by a self-splicing intron [49-51]. The introns will only splice out from a transcript generated by the L1 or CMV promoter. The spliced RNA is reverse-transcribed, followed by integration of the cDNA into the genome. The new insert contains a functional Blast gene. Blasticidin resistance will be obtained only if retrotransposition occurs. $\mathbf{b}$ and $\mathbf{c}$ Number of clones formed after puromycin and blasticidin selection. Blast ${ }^{R}$ foci were fixed to flasks and stained with Giemsa for visualization. Bars represent the mean blasticidin resistant colonies \pm standard deviation, shown as error bars for each construct

members of the L1D2 and L1D3 subfamilies. The four antisense 5'UTR were in the L1D1, L1D2, L1D3, and L1D7 subfamilies. Two 5'UTRs of active L1 s from the human genome and one 5'UTR of active L1 from the mouse genome were used as positive control; the schematics of these vectors are shown in Fig. 5a. The sense and antisense 5'UTRs of pig L1 s displayed lower or no promoter activity compared with human and mouse. Strong promoter activities were observed for all 5'UTRs of human and mouse in all four cell lines. 
Table 2 Number of ERV detected by LTRHarvest and Retrotector in the pig genome

\begin{tabular}{lll}
\hline Structure & Number of detected elements & Retrotector \\
\cline { 2 - 3 } & LTRHarvest & 2165 \\
\hline Total & 5456 & 290 \\
ERV containing RT (about 700 bp) & 20 & 80 \\
ERV containing gag (about 1500 bp) & 18 & 67 \\
ERV containing pol (about 3500 bp) & 12 & 30 \\
ERV containing env (about 3500 bp) & 9 & 19 \\
ERV containing gag, pol, and env & 19 & 2 \\
Copy number of Non-redundant FL ERVs & 2 & \\
Copy number of putative active ERV &
\end{tabular}

Three sense 5'UTRs (L1D1, L1D2, L1D7) and one antisense 5'UTR (L1D2) of pig L1 s showed detectable promoter activity (Fig. 5c).

\section{Young L1 $\mathrm{s}$ and ERVs displayed sense and antisense expressions in multiple tissues and cell lines}

The sense and antisense expressions of the youngest families from three types of retrotransposons, including L1D of L1 s, SINEA of SINEs, and ERV6 of ERVs, were evaluated by real-time qualification PCR (RT-qPCR) in 12 pig tissues (heart, liver, spleen, lung, kidney, duodenum, jejunum, brain, cerebellum, leg muscle, stomach, colon, testis, ovary) and two pig cell lines (PK15 and PEF). The primers were designed to target the conserved regions of 5'UTR, ORF1, and ORF2 of L1D1, and SINEA, and the conserved regions of LTRs, gag, pol, and env genes of ERV6 (Fig. 6a). The quality of RNA extracted from each sample was confirmed by RNA electrophoresis. RNAs treated with DNase and cDNAs were used as negative and positive control templates, respectively, for PCR amplification of ORF1 of L1 and gag of ERV to identify potential DNA contamination (data not shown). Overall, we found all types of detected young retrotransposons showed a similar expression profile between somatic tissues and cell lines; they all displayed antisense expression. Differential expression profiles across L1, SINE, and ERV retrotransposons were observed in the gonads (ovary and testis) (Fig. 6b-d). The sense expressions of L1 ORF1, L1 ORF2, ERV gag, ERV pol, and ERV env and the antisense expression of ERV LTR were repressed in the gonads, while clear antisense expression of L1 5'UTR was observed. In addition, both the sense and antisense transcripts of SINE were detected in the ovary, but neither were detected in the testis. The ORF1 and ORF2 of L1 displayed similar sense expression profiles in somatic tissues and cell lines, with high levels in the lungs and spleen, medium levels in the brain, cerebellum, colon, duodenum, kidney, liver, and stomach, and low levels in heart, jejunum, muscle, and PK15 and PEF cell lines. Antisense expression 5'UTR of
L1 in these tissues and cells displayed similar patterns to ORF1 and ORF2, but with medium or low levels (Fig. 6b). The expression pattern of SINE in different somatic tissues and cell lines was similar to that of LINE. The sense and antisense expressions of SINE had almost the same pattern (Fig. 6c). The antisense expression of ERV6 LTR and the sense expression of ERV coding regions (gag, pol, env) had similar overall profiles to those of LINE and SNIE, but higher antisense expression levels of ERV6 LTR were observed in the brain and cerebellum (Fig. 6d). Taken together, our data suggest that these retrotransposons may share a common regulatory mechanism in somatic tissues and cell lines, but a differential regulatory mechanism in gonads.

\section{Over $80 \%$ of protein coding and IncRNA genes overlap with retrotransposon insertions}

The intersection analysis between protein coding genes, lncRNA genes, their flank regions, and TE insertion positions indicated that the majority of protein coding and lncRNA genes overlapped with TE insertions. In general, $81.94 \%(17,278$ out of 21,087$)$ of the protein coding genes and $84.09 \%(12,174$ out of 14,477$)$ of lncRNA genes contained TE insertions (Fig. 7a), accounting for about $35.73 \%$ and about $8.25 \%$ of the total TE insertions, respectively (Fig. $7 \mathrm{~b}$ ). In detail, $79.27 \%$ of protein coding and $73.35 \%$ of lncRNA genes harboring SINE insertions, $71.26 \%$ of protein coding and $63.42 \%$ of lncRNA genes harboring LTR insertions, $69.95 \%$ of protein coding and $62.08 \%$ of lncRNA genes harboring LINE insertions were observed respectively (Fig. 7a). One-third of TEs hit the introns of protein coding gene $(35.10 \%$ of total TE insertions) and some hit lncRNA introns $(7.98 \%$ of total TE insertions), but very few ( $<1 \%$ of total TE insertions) were in the exons. Furthermore, a substantial proportion (5.91\%) of TE insertions hit the overlapping regions of protein coding and lncRNA genes (Fig. 7b). In addition, 9341 (44.30\%) protein coding genes and 3494 (24.13\%) lncRNA genes contained insertions from the youngest retrotransposon subfamilies, including L1s (L1D1-7), 


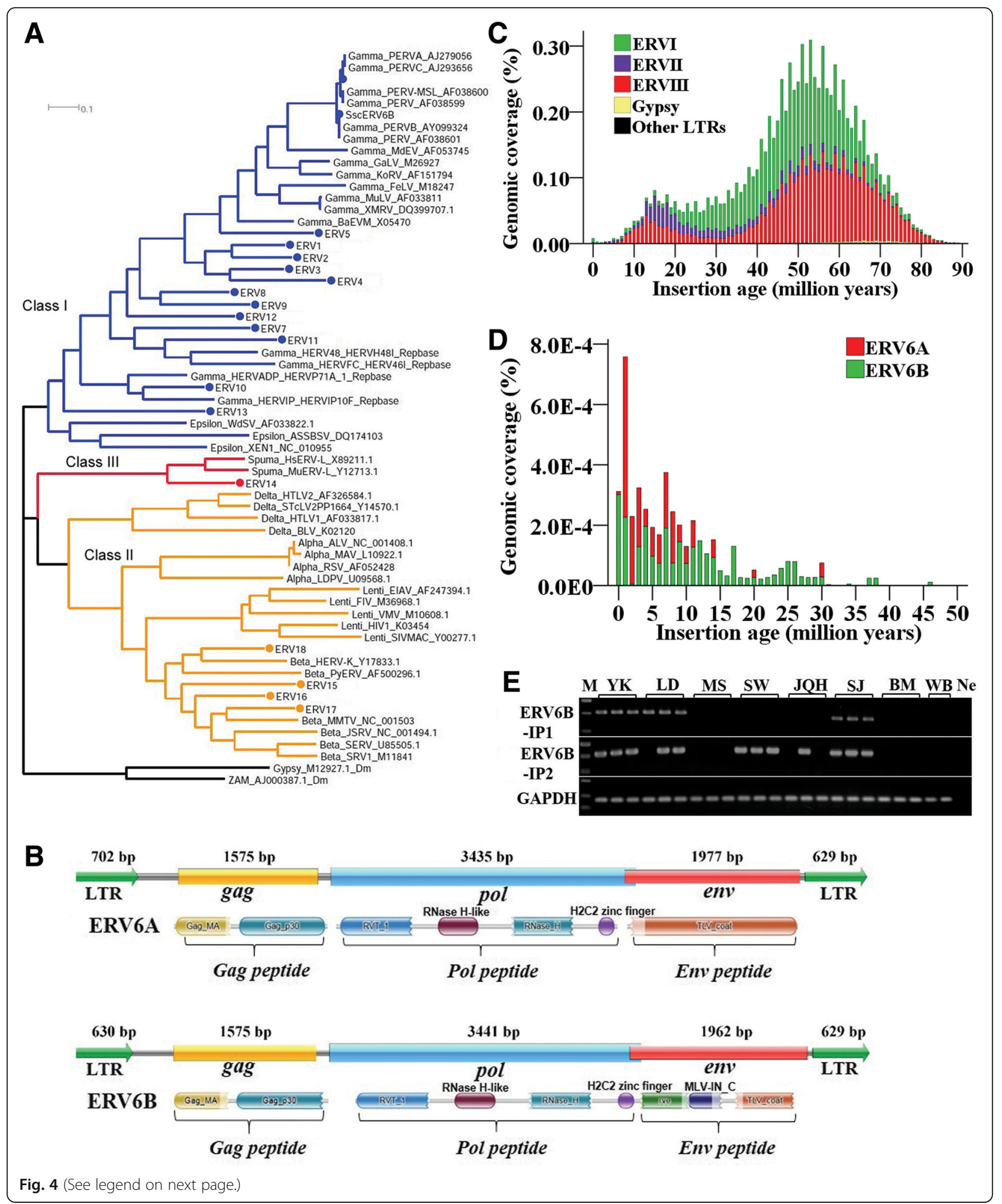


(See figure on previous page.)

Fig. 4 Evolution of ERVs in the pig genome. a ERVs were classified into 18 ERV families (ERV1-18) based on the phylogenetic tree inferred by using the Neighbor-joining method with the MEGA7 program, and the reference RT sequences from species other than pigs are included for comparison, shown with dots and described in the methods. b Structural schematics of the ERV6A and ERV6B, which featured LTR-gag-pol-envLTR and were presumed to be active. Gag_MA: Matrix protein (MA), p15; Gag_p30: Gag P30 core shell protein; RVT_1: Reverse transcriptase (RNA-dependent DNA polymerase); RNase H-like: RNase H-like domain found in reverse transcriptase; rve: Integrase core domain; MLV-IN_C: Murine leukemia virus (MLV) integrase (IN) C-terminal domain; TLV_coat: ENV polyprotein (coat polyprotein) (c) Age distribution of pig ERV classes. d Age distribution of the youngest pig ERV subfamilies (ERV6A and ERV6B). e Insertion polymorphism detection of the youngest pig ERV subfamilies (ERV6B) by PCR. Breed name abbreviations are the same as those in Fig. 1f. The $x$-axis represents the insertion age (Mya), and the $y$ axis represents the percentage of the genome composed of retrotransposon families/subfamilies (\%) in Fig. $\mathbf{c}, \mathbf{d}$

A

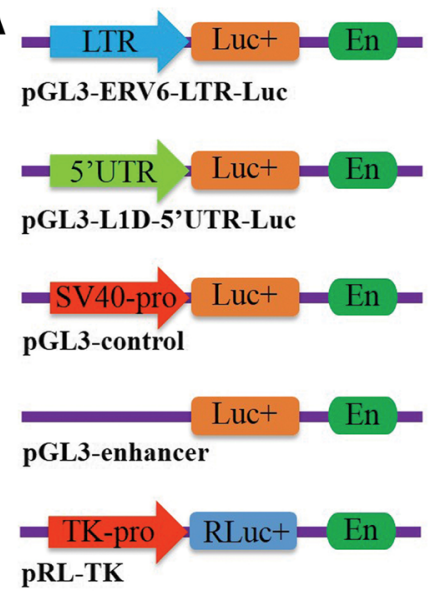

C

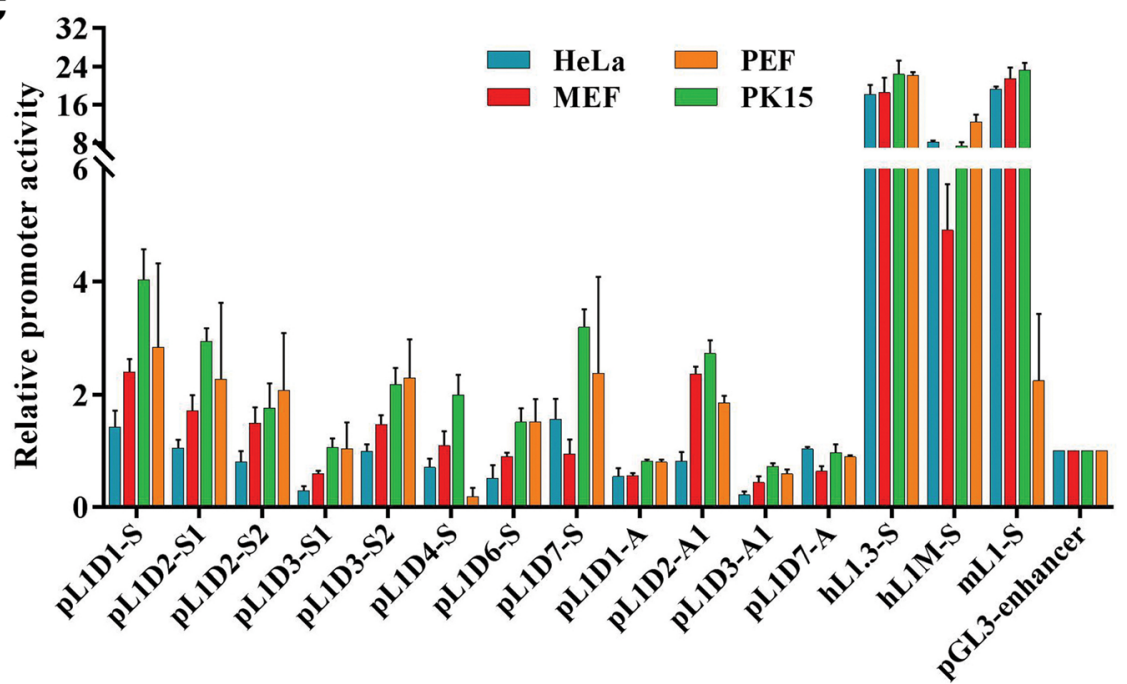

Fig. 5 Sense and antisense promoter activities of pig L1 5'UTRs and ERV6 LTRs. a Schematics of vectors used for promoter activity detection by luciferase assay. The sense and antisense $5^{\prime} \mathrm{UTR} / \mathrm{L} 1$ and LTRs of ERVs from young and putatively active subfamilies of L1 were cloned into the pGL3-enhancer luciferase reporter vector to investigate the promoter activity. $\mathbf{b}$ Sense and antisense promoter activities of ERV6A and ERV6B LTRs measured by luciferase assay. c Sense and antisense promoter activities of young L1 5'UTRs (L1D) measured by luciferase assay. Eight sense and four antisense L1 5'UTRs from different subfamilies of L1D family were cloned as described in the methods, and two 5'UTRs (hL1-3 and hL1-M) of active L1 s from human and one $5^{\prime} \mathrm{UTR}(\mathrm{mL} 1)$ of active L1 from mouse were used as positive controls

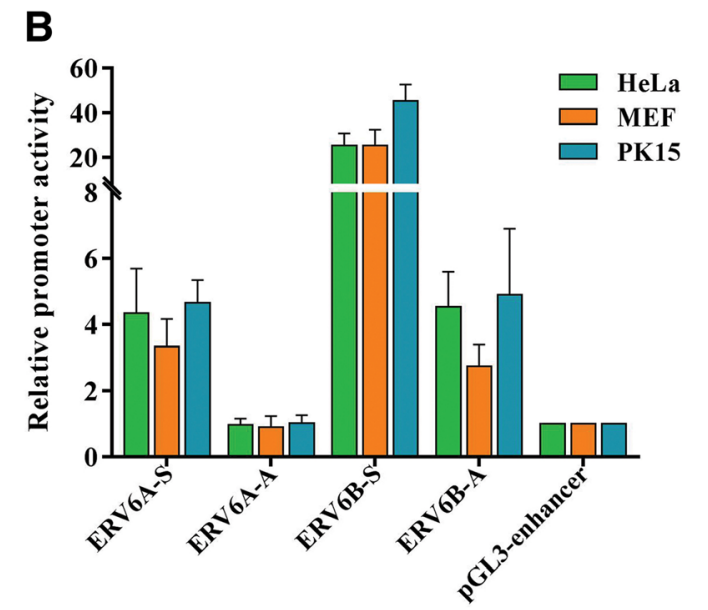




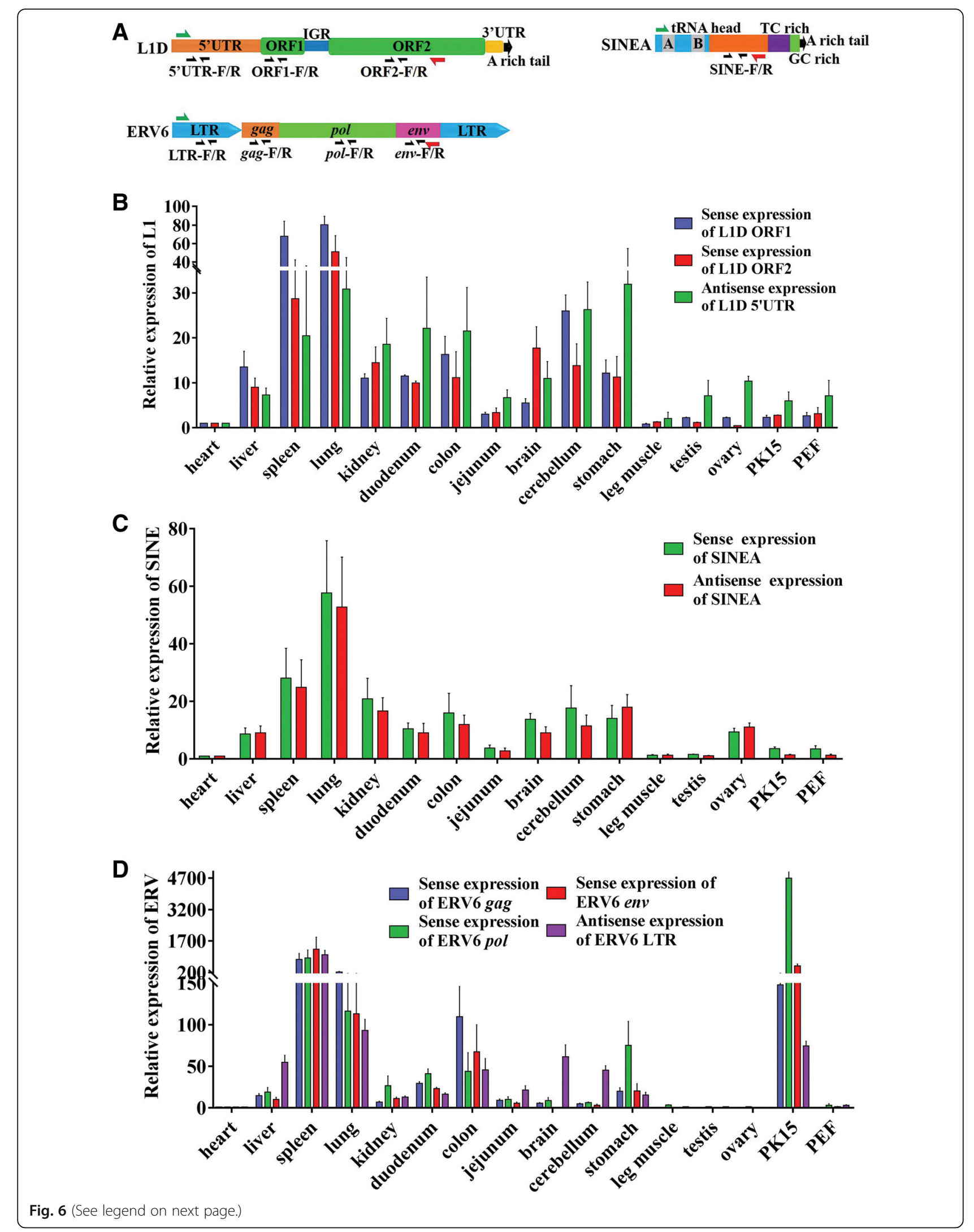


(See figure on previous page.)

Fig. 6 Sense and antisense expression profiles of pig L1D of L1 S, SINEA of SINES, and ERV6B of ERVs. a Primer design for reverse transcription (RT) and real-time quantitative PCR (RT-GPCR) detection. The primer for sense and antisense RT are indicated by red and green arrowheads, respectively, and the primers of ORF1-F/R, ORF2-F/R, 5'UTR-F/R, pol-F/R, gag-F/R, env-F/R, LTR-F/R, SINE-F/R (black arrowheads), are used for RT-qPCR to detect the expression of 5'UTR, ORF1, and ORF2 of L1, LTR, gag, pol, and env of ERV6 and SINE, respectively. $\mathbf{b}$ Sense expression of ORF1 and ORF2, and antisense expression of 5' UTR of L1D in tissues and cells. c Sense and antisense expression of SINEA in tissues and cells. $\mathbf{d}$ Sense expression of gag, pol, and env of ERV6, and antisense expression of LTR of ERV6 in tissues and cells

SINEs (SINEA1-3), and ERVs (ERV6A and ERV6B). The youngest SINE subfamilies (SINEA1-3) displayed the most extensive distribution in protein coding (9230/ $43.77 \%)$ and lncRNA (3402/23.50\%) genes, and represented the highest insertion frequency compared with other retrotransposon types (Table 3).

While the annotation of the mobilome in pig revealed that young retrotransposon subfamilies only occupied a small proportion of the pig genome, with less than $1 \%$ of total genome covered by the youngest subfamilies (L1D1-7/0.19\%, SINEA1-3/0.63\%, and ERV6/0.02\%) (Additional file 2: Figure S4A), compared with the genome coverages of LINE (18.52\%), LTR (7.56), and SINE (11.05\%) (Additional file 2: Figure S4B). The pig-specific L1 s, SINEs, ERVs represented about 10.00, 10.00, and $7.00 \%$ of the genome, whereas the youngest subfamilies of L1 (L1D) and SINEA represented 1.13 and $7.64 \%$ of the genome, respectively (Additional file 2: Figure S4C). In addition, lncRNA and protein coding genic regions and their flank regions exhibited many biases in their retrotransposon composition and orientation relative to genomic averages (Fig. 7c and Additional file 2: Figure S4D). Though also the most prevalent TE families in the genic regions of IncRNA and protein coding genes, and their flank regions, LINEs were significantly depleted, with a range from 11.79 to $14.88 \%$ in protein coding and lncRNA genic, and their flank regions, with the exception of LINEs in lncRNA genic regions, where the LINEs represented similar coverage to the genomic average of about $18 \%$. Both 5' - and 3'-flanks of lncRNA and protein coding genes tended to slightly enrich SINEs compared with their genic regions and genomic average, respectively (Fig. 7c). Most retrotransposons tended to insert into the opposite orientation in introns and exons of both protein coding and lncRNA genes; in particular, more than 30 and $15 \%$ of LINEs inserted in the opposite orientation in the introns of protein coding and lncRNA genes. A significant difference of insertion orientation frequency was observed for LINEs in introns of protein coding genes $(p<0.05)$, while SINEs in exons of protein coding genes and LTRs in exons of lncRNA genes displayed a bias of sense insertion orientation. The bias of sense insertion orientation of SINEs in exons of protein coding genes was also well supported by EST dataset analysis (Additional file 2: Figure S4D).

\section{Significant contribution of retrotransposons to the transcripts of IncRNA and protein coding genes}

Intersection analysis showed that lncRNAs tended to enrich the TE-derived sequences compared with mRNAs, and nearly half $(46.66 \%, 13,804 / 29,585)$ of lncRNAs overlapped with at least one TE (Fig. 7d), and 4.42\% $(1307 / 29,585)$ of lncRNAs designated as retrotransposon-lncRNAs, where more than $70 \%$ of the whole lncRNAs were covered by retrotransposons. In fact, about 15\% of lncRNA sequences were occupied by TEs (Fig. 7e). By contrast, TEs overlapped only $28.50 \%$ of mRNAs, and covered only $5.30 \%$ of mRNA sequences (Fig. $7 \mathrm{~d}$ and e). Retrotransposons were the major contributors of pig lncRNAs; they overlapped $43.04 \%$ of lncRNAs and covered $12.78 \%$ of their sequence (Fig. $7 \mathrm{~d}$ and e). Furthermore, lncRNAs exhibited many biases in their TE composition relative to genomic averages. Though the LINEs and SINEs were the most prevalent in the pig genome, accounting for 18.52 and $11.05 \%$ of genomic sequences, respectively, both L1 s and SINEs are significantly depleted by about 4.0-fold. Conversely, LTRs are slightly enriched in lncRNAs compared with other retrotransposon types (Fig. 7e).

Almost half of the protein coding genes (45.83\%) could generate chimeric transcripts with TEs (Fig. 7f). Retrotransposons were the major contributors of these chimeric transcripts, and they accounted for $4.89 \%$ of mRNA sequence; in total, $26.62 \%$ of mRNAs and $1.64 \%$ of ESTs contained retrotransposon-derived sequences, and these transcripts corresponded to $43.78 \%$ of protein coding genes (Fig. 7d and f). In addition, mRNAs comprised nonrandom distribution of retrotransposons, and SINEs were the most prevalent in mRNAs, overlapping with $21.03 \%$ of mRNAs and $1.05 \%$ of ESTs, respectively, accounting for $2.28 \%$ of mRNA sequences and corresponding to $34.84 \%$ of total protein coding genes. LINEs and LTRs only overlapped with about $10 \%$ of mRNAs and $0.4 \%$ of ESTs, respectively, and accounted for 1.10 and $1.51 \%$ of mRNA sequences, respectively, which corresponded to about $20 \%$ of protein coding genes (Fig. $7 \mathrm{~d}$ and $\mathrm{f}$ ). In addition, retrotransposons, mainly represented by SINEs, were primarily located in 3'UTRs of mRNAs, and overlapped $28.38 \%$ of 3'UTRs of mRNAs; this bias of SINEs was not observed for the 3'-end of IncRNAs (last exon). Conversely, less than $0.4 \%$ CDS and $3.64 \%$ of 5'UTRs overlapped with TEs, and there appeared to be 

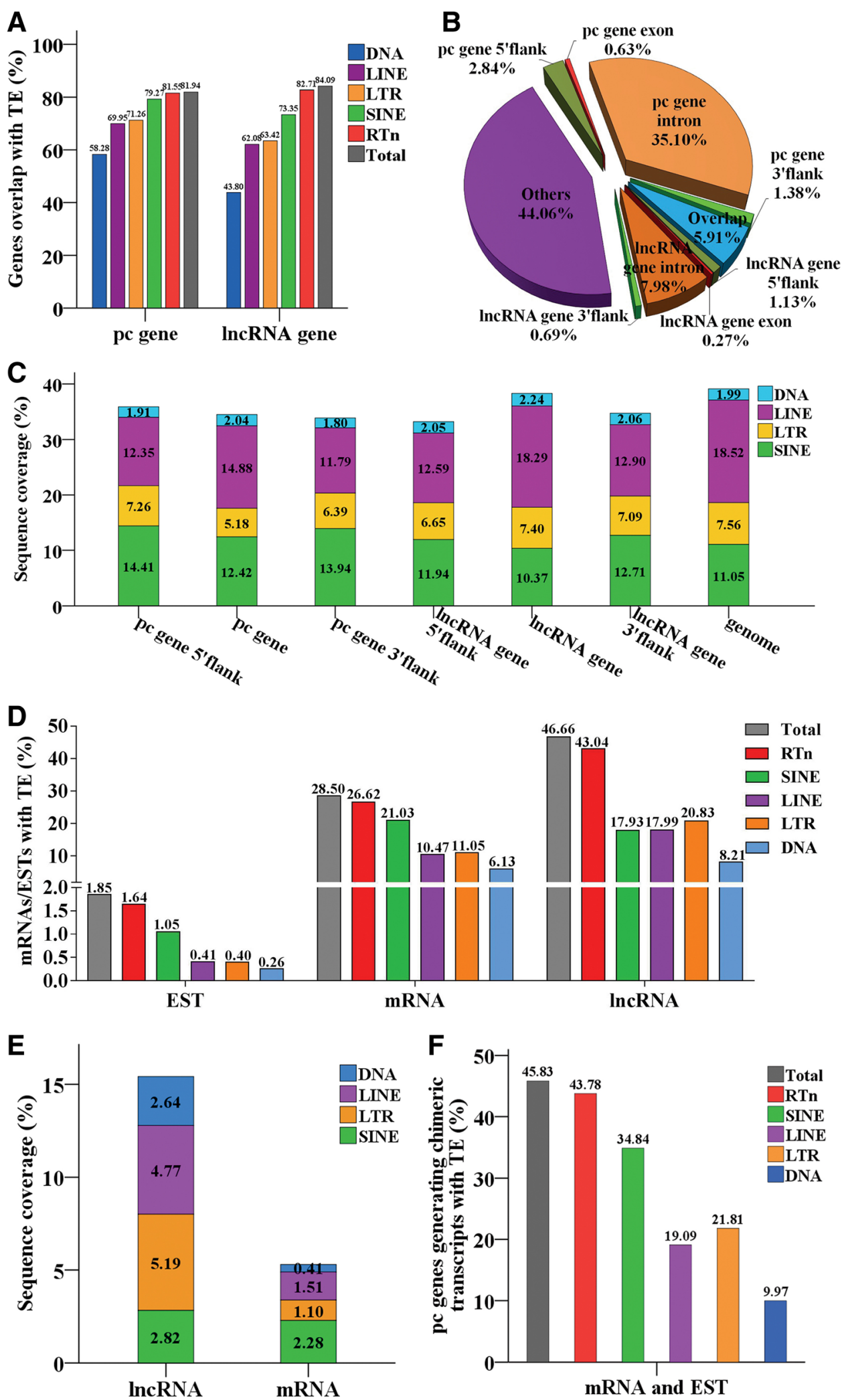

Fig. 7 (See legend on next page.) 
(See figure on previous page.)

Fig. 7 Retrotransposons contribution to protein coding and IncRNA genes. a The proportion of protein coding (pc) genes and IncRNA genes overlapping with retrotransposon insertions. $\mathbf{b}$ The proportion of TE insertions in the introns and exons of protein coding and IncRNA genes, and their flank regions. c The genomic coverage of retrotransposons in protein coding (pc) and IncRNA genic regions, and their flank regions. $\mathbf{d}$ The proportion of mRNAs, ESTs, and IncRNAs containing retrotransposon-derived sequences. e Sequence coverage of retrotransposons in IncRNAs and mRNAs. $\mathbf{f}$ The proportion of the protein coding genes generating chimeric transcripts with retrotransposons

significant deletion of TE-derived sequences (Additional file 2: Figure S4E).

\section{Discussion}

Both L1 s and SINEs displayed multiple wave amplifications dominated by different families in the evolution of the pig genome

The data presented here defined the classification of major retrotransposon types (L1 s, SINEs, and ERVs) at multiple levels, and the evolution dynamics analysis revealed that these retrotransposons presented multiple wave amplifications that were dominated by different families in the evolution of the pig genome. We classified pig-specific L1 s into four distinct families (L1A, L1B, L1C, and L1D) and 51 subfamilies. About 100 L1 copies were identified as intact and putatively active elements, which is similar to that in human, where it was postulated that out of the 1318 full-length L1 sequences, 146 were intact and putatively active, but substantially lower in number compared with that in mice, where 2811 out of 14,076 full-length L1 elements were estimated to be potentially retrocompetent [40].

Table 3 The number of IncRNA genes and protein coding genes contain the insertions from youngest retrotransposons

\begin{tabular}{lll}
\hline Young RTn & protein coding gene & IncRNA gene \\
\hline L1D1 & 120 & 49 \\
L1D2 & 129 & 60 \\
L1D3 & 47 & 25 \\
L1D4 & 61 & 29 \\
L1D5 & 149 & 59 \\
L1D6 & 108 & 28 \\
L1D7 & 204 & 96 \\
Total youngest L1 s & $660(3.13 \%)$ & $286(1.98 \%)$ \\
ERV6A & 30 & 13 \\
ERV6B & 24 & 17 \\
Total youngest ERVs & $42(0.20 \%)$ & $21(0.15 \%)$ \\
SINEA1 & 3464 & 1038 \\
SINEA2 & 7696 & 2612 \\
SINEA3 & 3786 & 1134 \\
Total youngest SINEs & $9230(43.77 \%)$ & $3402(23.50 \%)$ \\
Total & $9341(44.30 \%)$ & $3494(24.13 \%)$ \\
\hline
\end{tabular}

The percentage in parentheses is the percentage of protein coding/IncRNA genes with youngest retrotransposon insertions account for total protein coding/IncRNA genes
Substantially different amplification dynamics of families of L1 s during the evolution history of the pig genome were observed. Ancestral pig genomes contained two distinct L1 families (L1A and L1B), which amplified and evolved simultaneously for about 80 million years, ranging from 85 Mya to 5 Mya. Then, two families (L1C and L1D) replaced their predecessors as the dominant families, and these were amplified over the last 20 million years. The four families occasionally coexisted in pig evolution for a short period of between 20 Mya and 5 Mya. This pattern of evolution was generally similar to that of humans, where several old and distinct L1 families coevolved for over 30 million years in the ancestral genome, and a new family of L1 amplified over the last 40 million years [53]. The families of L1A, L1B, and L1C were old and more divergent than that of L1D. The former showed no sign of current activity because of extensive accumulated mutations, whereas the L1D family represented the most active family of L1, which was also supported by the identification of about 100 intact L1 elements and the insertion polymorphisms of this family in both inter- and intra-breed pigs. These data suggest that L1D represented the most active family of L1 in pigs. Pig-specific SINEs, with a length between 102 and 265 bp (without polyA tail, Additional file 2: Table S2), were classified into three families (SINEA, SINEB, and SINEC) based on sequence similarity and length; the three families display periodic fluctuations with three large waves of fixation, and occasionally coevolved for a long period between 20 Mya and 80 Mya. SINEB and SINEC are old families, and the activity was extinct in the last 20 million years, while the SINEA family represented the most recent expansion and still displayed activity during the last 10 million years; SINEA1-3 represented the youngest subfamilies of this family. These data indicate that both L1s and SINEs displayed periodic fluctuations with multiple wave amplifications, but were dominated by different families in the evolution of the pig genome, and some families of both L1 s and SINEs coevolved at particular stages.

\section{ERV6s are "modern" ERVs}

ERVs, which are the dominant LTR retrotransposons within mammalian genomes, have been invading mammalian lineages for over 100 million years [54]. Early genome sequencing studies showed differences in the activity of retroviruses among mammalian species, with humans largely containing inactive ERV families [6] and 
mice containing numerous active ERV families [29]. These active ERVs are generally referred to as "modern" ERVs because they have integrated into the host genome after speciation and are closely related to exogenous viruses. They are still able to produce infectious viruses because of the lack of deactivating mutations. Active ERVs have also been found in other mammal species, such as in koala (KoRV) [55], Jaagsiekte sheep (JSRV) [56], and domestic cat (ERV-DC) [57]. In this study, we characterized the diversity, structure, activity, and evolutionary history of pig ERVs. Thousands of ERV candidates were identified in the present study, and most of these ERVs had decayed; only about 250 candidates contained intact RT regions, which were classified into 13 gamma ERVs, three beta ERVs, and one spuma ERV by phylogenetic analysis. Moreover, most of these families appeared to be more defective, with a striking deceleration in recent activity, with the exception of ERV6, which belonged to the gamma retroviruses of ERVI and included two subfamilies (ERV6A and ERV6B); this family still exhibited an extended period of expansion and showed signs of increased activity in the last 10 million years, with a few copies encoding long peptides with intact gag, pol, and env domains, which is in good agreement with the findings in the Wuzhishan pig genome [45]. Furthermore, all the active pig ERVs $(\gamma 1 \mathrm{~A}, \gamma 1 \mathrm{~B}$, and $\gamma 1 \mathrm{C})$ reported in previous studies [52] were also classified into this family. The ERV6B subfamily tended to be the youngest and most active subfamily based on age analysis, and the insertion polymorphisms of this subfamily were also confirmed. Overall, these data suggest that most ERVs are fossils that are fixed in the pig genome, while ERV6s are "modern" ERVs that are putatively active and play a role in the evolution of the genome. In addition, theses ERVs carry potential risks for human xenotransplantation, which have been extensively noted $[58,59]$.

\section{Evidence for sense and antisense promoter activities of L1 5'UTRs and ERV LTRs}

The insertions of retrotransposons may impact gene activity by offering alternative RNA polymerase II (Pol II) promoters. It seems that most retrotransposons harboring Pol II promoters, such as ERVs, often contained RNA polymerase II (Pol II) promoters within the LTR flanking coding sequence of the elements [60]. Both sense and antisense Pol II promoter activities of L1 $\mathrm{s}$ in humans and mice have been characterized. Moreover, the antisense coding capacity of human L1 has been established [61]. The antisense Pol II promoter of human L1 is located in the 5'UTR, while that of mice is located in the ORF region [62]. It has been confirmed that the L1 antisense promoter activity could drive chimeric transcripts [36, 63]. In the present study, we provided evidence to support the sense and antisense Pol II promoter activities in the 5'UTRs of pig L1 s, although the activity levels were low compared with those in humans and mice. The sense Pol II promoter activities of pig ERV have been characterized [64, 65], and our data confirmed the sense Pol II promoter activities of both ERV6A and ERV6B subfamilies. This was the first time we observed the antisense Pol II promoter activities of ERV6. The promoter activity analysis of these young retrotransposons offers a new perspective to understand their impact on genome, given that new insertions can provide new promoters. Such examples of host genes driven by TE promoters have been documented in diverse species over the past several decades [66-68]. Generally, TE promoters often show spatially or temporally regulated activity that is dependent on cell type and/or in response to environmental cues such as stress or infection $[69,70]$.

In addition, the detected young retrotransposons showed similar overall sense and antisense expression profiles in somatic tissues and cell lines in the current study, indicating that these retrotransposons may share a common regulatory mechanism in somatic tissues and cell lines. However, in the gonads (ovary and testis), their expression patterns are different, indicating different regulatory mechanisms. A lack of sense expressions of L1 and ERV, and obvious antisense expression of L1 5'UTR, was observed in the gonads, which supports previous studies, but also suggests that their expression may be restricted to various stages of gametogenesis [60]. Germline suppression of TE activity can be achieved through both the epigenetic mechanism, including DNA methylation and heterochromatin formation, and small RNA-mediated post-transcriptional regulations $[71,72]$. The antisense expression of L1 may actually play a role in the repression of sense expressions of L1 in the gonads by an RNA interference pathway, as suggested previously [62, 73]. However, the activation of sense and antisense transcriptions of SINE in the ovaries is very interesting and suggests a biological role of SINE in this specific tissue, which may warrant further study to elucidate its physiological significance. On the other hand, the sense and antisense transcripts of these retrotransposons detected by qPCR in current study may not only originate from TE's own promoters, but also generate from host gene promoters by co-expression (fusion expression) or other expression ways since the overlapping of retrotransposons and host genes is very common in genome.

\section{Retrotransposition competence of pig L1}

We demonstrated that one subfamily of the youngest L1 s (L1D1) in pigs is capable of mobility by retrotransposition assay, which was also well supported by previous study, where it was found that a recent full-length endogenous L1 insertion in KPL2 gene caused the infertility of Yorkshire boars [74]. The retrotransposition activities of young L1 and SINE (Alu) in humans and L1 and SINE (B1) in 
mice have also been proven experimentally [41]. These data further suggest that most mammals contain retrocompetent L1 and SINE. In addition, the retrotransposition activities of pig L1 were cell-specific, with high activity in human HeLa cells and very low activity in pig PK15 cells, while human L1 was not mobile in this cell line. Compared with human L1, the detected pig L1 (L1D1) displayed lower levels of retrotransposition activity, which could be an indication of either the low activity of the cloned element or the low overall retrotransposition activities of all pig-specific L1 s. In fact, the promoter activities of most detected young pig L1 subfamilies were lower than those of humans and mice in the present study, which supports the latter possibility. However, we also couldn't exclude the possibility is that the retrotransposition of pig L1 may need additional cellular factors, and the retrotransposition assay need to be optimized in pig PK15 cells. As suggested by others, different components of L1, including 5'UTR, ORF1 and ORF2, and IGR, may impact the activity of L1 [43]; here, we also found that IGR plays an important role in the retrotransposition of L1. The retrotransposition activity of pig L1 was improved significantly with the replacement of human IGR, a finding that was also found in bat L1 [43], indicating that IGR plays a role in the evolution of L1. In addition, the risk of cross-species transmission of pig ERVs has been a concern in xenotransplantation [59]; here, our data provided experimental evidence of the retrotransposition-competent nature of pig L1 in human HeLa cells, indicating that the active pig L1 $s$ and SINEs also carry a potential risk of horizontal transfer in xenotransplantation, which warrants further evaluation.

\section{Deep impact of retrotransposons on IncRNA and protein coding genes}

Mammals are the best-studied vertebrates, largely because of the higher number of sequenced genomes spanning major lineages within the group [75]. Here, we found that the composition of TEs in the pig genome is dominated by retrotransposons, with LINE, LTR, and SINE accounting for $18.52,7.56$, and $11.05 \%$ of the sequenced genome, respectively, representing the typical mammalian characteristics $[6,29,42]$. However, the coverage of total repeat contents $(40.72 \%)$ by this study is similar to that in early TE annotation of Duroc genome [30], but higher (38.2\%) than that in Wuzhishan genome [45]. This disagreement may be due to an underestimation, since the Wuzhishan genome is far from complete compared with the reference genome of Duroc and dense repeat regions are underrepresented in the previous draft assembly. The high coverage of TEs in the genome and their ability to re-infect or move within the genome gives TEs an intrinsic propensity to possibly affect host genes. A significant association between the presence of intragenic L1 s and down-regulated genes in early embryogenesis was found in humans and mice [76]. L1 elements were present in an estimated 79\% of human genes in at least one copy [6]. There are at least 124 documented LINE1-mediated insertions that have resulted in genetic disease in humans [77]. Many phenotype variations due to TE insertions have been observed in animals, such as SINE insertion causing body size variation and coat color pattern change in dogs [78-80], and ERV insertion causing eggshell color variation in chickens [81]. Two cases of phenotype variations due to L1 insertion were observed in pigs [82, 83].

Here, our data demonstrated that retrotransposons have an extensive impact on lncRNA and protein coding genes at both the genomic and transcriptomic levels. In pigs, 35.73 and $8.25 \%$ of the total TE insertions overlap with protein coding and lncRNA genes, respectively, and about $80 \%$ of protein coding and lncRNA genes contain retrotransposon insertions, which is generally similar to the estimations (about 90\%) of the protein coding genes of bovines [84], mice, and humans [85, 86]. In addition, we found that although the youngest retrotransposons, including L1D1-7 of L1 s, SINEA1-3 of SINEs, and ERV6 of LTRs, occupy less than 1\% of the genome; they overlap with about half of protein coding genes $(44.30 \%)$ and one-fourth $(24.13 \%)$ of lncRNA genes. These insertions may be new mobilization events, and the insertion polymorphisms of these families/subfamilies were also confirmed in the current study, indicating that the insertions of young retrotransposons may contribute to the structure variations of these genes, or even gene activities. These data also indicate that the retrotransposon insertion polymorphisms may be a very useful genetic marker to develop and warrants further study.

The intersection analysis between retrotransposon insertions and transcripts (ESTs and mRNAs) of protein coding genes revealed that at least $40 \%$ of protein coding genes are estimated to generate chimeric transcripts with retrotransposons, which are generally similar to the estimations in humans and mice, where $39 \%$ of humanand $35 \%$ of mouse-specific exons overlap with retrotransposons [87]. Retrotransposons are believed to be closely associated with the birth, evolution, expression, and function of lncRNAs in mammals, and strong contributors of lncRNAs [88, 89]. A significant negative correlation between the content of TEs and the level of expression of IncRNAs was observed [26, 28]. Very recently, a new class of natural lncRNAs that can activate translation by targeting sense mRNAs through the activity of embedded inverted SINEB2 elements, called SINEUPs, has been well characterized in mammals [90, 91]. The modular organization of SINEUPs strongly suggests that embedded TEs are fundamental for lncRNA function. This study also confirmed that the pig lncRNAs tend to enrich TE-derived sequences compared with mRNAs, which generally agrees with the findings in 
other mammals (including humans and mice) and fish (zebrafish) [26, 27]; however, the proportion (46.6\%) of lncRNAs overlapping TEs in pigs is substantially lower than that in humans (83.4\%), mice (68.2\%), and zebrafish $(66.5 \%)[26,27]$. This may be due to species differences; however, it clearly shows the importance of TE for IncRNA evolution. TE-derived sequences in the pig lncRNAs are dominated by retrotransposons, which overlap $43.04 \%$ of lncRNAs and cover $14.37 \%$ of their sequences; the same trend was also observed for the lncRNA sets in humans and mice [27, 28], suggesting that the high content of retrotransposon sequences is likely a contributing factor to sequence diversification and that the high complexity of lncRNAs is a general property in mammals.

In addition, significant biases in retrotransposon composition, orientation, and location in lncRNA and protein coding genes and their transcripts were observed. The mammal genomes are largely dominated by LINEs [6, 29, 30]. However, the most striking departure from this general trend is apparent in pig lncRNA and protein coding genic regions and their flanking regions. LINEs seem underrepresented in these regions, with the exception of the IncRNA genic region, which generally agrees with the trends in humans and mice [28]. In addition, LINEs and LTRs tend to insert in an antisense orientation in the introns of protein coding and lncRNA genes in pigs, and a similar trend of LINEs and LTRs has also been observed in the introns of protein coding genes of bovines [84] and humans [87]. Most retrotransposons (LINEs, LTRs, and SINEs) in the exons of protein coding and lncRNA genes in pigs are also preferentially inserted in the opposite orientation, whereas SINEs in exons of protein coding genes display a significant bias of sense insertion orientation, supporting the observations in bovine [84] and human studies [92]. LTRs in pig lncRNA exons also display sense orientation insertions, which is similar to humans [26], whereas SINEs tend to be enriched in the 3 '-end of lncRNAs, and appear more often in the sense orientation in humans [26]; these biases of SINEs were not observed in pig lncRNAs. Furthermore, pig mRNAs and lncRNAs exhibit many biases in their retrotransposon composition and location. A relative under-repression of LINEs and SINEs, and slight enrichment of LTRs in pig lncRNAs were observed, similar to humans and mice $[27,88]$, whereas retrotransposons are primarily located in 3'UTRs of pig mRNAs; they were rarely located in 5'UTRs and coding regions. mRNAs, mainly represented by the 3'UTRs of mRNAs, tend to enrich SINEs other than LINEs and LTRs, also similar to humans and mice [92-94]. Global expression data indicate that the retrotransposon sequences in the 3 'UTRs negatively affect the expression of mRNAs [93], suggesting that the SINEs in 3'UTRs may serve as targets for microRNAs $[95,96]$, thereby supporting another biological role of SINEs in the 3'UTRs of mRNAs.
In summary, these data indicate that redistribution of retrotransposons is a general property of mammalian lncRNA and protein coding genes and their transcripts. Retrotransposons in mammal genes may share a common regulation mechanism during evolution, and retrotransposons also play an important role in the structural organization, evolution, expression, and function of both protein coding and lncRNA genes.

\section{Conclusions}

In the present study, we characterized the classification and evolution profile of retrotransposons in pigs. L1 s were detected and classified into four distinct families (L1A, L1B, L1C and L1D) and 51 subfamilies, and demonstrated that one youngest L1 s subfamily (L1D1) in pigs is capable of mobility by retrotransposition assay. SINEs were classified into three families (SINEA, SINEB, and SINEC) based on length and structure. ERVs were classified into 18 families (ERV1-ERV18) and most of ERVs had decayed, only ERV6 showed signs of increased activity in the last 10 million years, with a few copies encoding long peptides with intact gag, pol, and env domains. The sense and antisense expression profiles and promoter activities of young retrotransposons were characterized, young L1 5'UTRs and ERV LTRs displayed sense and antisense promoter activities. And we also investigated their impact on lncRNA and protein coding genes by defining the mobilome landscapes at the genomic and transcriptomic levels, significant distribution bias of retrotransposon composition, location, and orientation in IncRNA and protein coding genes, and their transcripts, were observed. These findings help provide a better understanding of retrotransposon evolution in mammal and their impact on the genome and transcriptome.

\section{Materials and methods \\ Retrotransposons Mining in the pig Genome}

The de novo detection of the L1 s in the pig genome was conducted with the MGEScan-non-LTR program [97]; however, most of the elements identified by MGEScannon-LTR were incomplete. To obtain the full length of the elements, the sequences identified with the MGEScannon-LTR program were aligned to the pig genome again by using Blat [98] $(-$ minIdentity $=100,-$ minScore $=200)$. The alignment result file was converted into bed format file, and an additional 2500 bp $5^{\prime}$-flanking sequences and 200 bp 3'-flanking sequences were extended for each L1 sequence to define the boundaries of 5'UTR and 3'UTR by using the bedtools slop command (-s, $-12500,-\mathrm{r} 200)$. In addition, the available pig L1 elements in the L1Base database [40] (http://l1base.charite.de/l1base.php) were also downloaded with a bed file format. These two datasets were merged and the redundancy was removed (loci distance within 3000 in the same strand). Finally, the 
sequences of these L1 elements with unique positions in the pig genome were extracted by using the bedtools getfasta command (bedtools v2.27.0). The boundary of these L1 elements were defined by alignment and then clustered based on the 5'UTR sequence similarity; any clusters with fewer than 10 elements were removed. The final consensus sequence was constructed by using cons in EMBOSS explorer (http://www.bioinformatics.nl/emboss-explorer/) for each L1 cluster. The 5'UTRs of each consensus sequences were used for subsequent phylogenetic analysis.

ERVs were identified with LTRharvest [99] and RetroTector [100]. The LTR nucleotide similarity threshold used in LTRharvest was $>80 \%$, with other parameters set to their defaults. A cutoff of 250 was used for RetroTector scores, as the majority of the elements with scores between 250 and 300 showed a conserved structure. Only ERVs with intact RT regions (about $0.5 \mathrm{~Kb}$ ) were retained and used for subsequent phylogenetic analysis and family classification. The consensus sequences or representative sequences were derived for each family/subfamily based on the phylogenetic tree.

\section{Phylogenetic analysis}

Multiple alignments were constructed from the DNA sequences of the 5'UTR of L1 and the RT regions of ERV retrotransposons by using the ClustalX2 [101] program, respectively. We chose to use the DNA sequences to make the multiple alignments and build the phylogenetic tree, rather than the amino acid sequence, because of the presence of numerous frame-shift mutations and stop codons in the ancient retrotransposon elements. A Neighbor-Joining tree was generated from the alignment by using MEGA7 [102] with Kimura 2-parameter model and complete deletion as parameters. Bootstrap values were obtained from 100 replicates. The reference RT sequences of ERVs from species other than pigs were included for defining the classification of pig ERVs. The GenBank accession numbers and abbreviations of ERVs used for phylogenetic analysis are as follows: FeFV, feline foamy virus (AJ223851); HFV, human foamy virus (Y07725); HIV-1, human immunodeficiency virus 1 (K03454); SRV-1, simian SRV-1 type D retrovirus (M11841); MMTV, mouse mammary tumor virus (NC_001503); RERV, rabbit ERV (AF480925); RSV, rous sarcoma virus (AF052428); BLV, bovine leukemia virus (K02120); FELV, feline leukemia virus (M18247); KoRV, koala type $\mathrm{C}$ endogenous virus (AF151794); MDEV mus dunni endogenous virus (AF053745); and MuLV, Moloney murine leukemia virus (AF033811). In addition, $\gamma 1 \mathrm{~A}$ (AJ279056), $\gamma 1 B$ (AY099324), and $\gamma 1 \mathrm{C}$ (AJ293656) are the porcine ERVs identified previously.

\section{Retrotransposon annotation in the pig genome and transcriptome}

The pig (Sscrofa11.1) genome was downloaded from the UCSC database (http://hgdownload.soe.ucsc.edu/goldenPath/susScr11/bigZips/). The lncRNA transcripts $(29,585)$ and their coordinates of IncRNA genes (Bed format file) were downloaded from the NONCODE database (http:// www.noncode.org/download.php). The Bed format file of lncRNA genes, which represents 17,811 lncRNA genes and corresponds to Sscrofa10.2, were converted into Sscrofa11.1 by LiftOver (http://genome.ucsc.edu/cgi-bin/ hgLiftOver), and finally, the coordinates of 14,477 lncRNA genes were obtained. The coordinates of protein coding genes $(21,087)$ and exons, the mRNAs $(45,788)$ of protein coding genes, and the $5^{\prime}$ UTR, 3 'UTR, and CDS of protein coding genes were identified from the annotation of Sscrofa11.1 in Ensembl (ftp://ftp.ensembl.org/pub/release91/gff3/sus_scrofa/). The total EST sequences $(1,676,489)$ and their genomic coordinates were downloaded from the EST database (https://www.ncbi.nlm.nih.gov/nuccore) and the UCSC database (http://genome.ucsc.edu/cgi-bin/hgIntegrator), respectively. The $5^{\prime}$ - and 3 '-flank coordinates of protein coding and lncRNA genes were constructed based on the genes by extending $5 \mathrm{~kb}$ and $3 \mathrm{~kb}$. The sequences of genes and flanks of genes were extracted from the genome by using bedtools getfasta according to their coordinates.

The newly identified L1 and ERV elements were combined to the known repeats in the pig genome, including SINEs from Repbase (version 20,170,127), and redundancies were filtered out to create a custom library. The distributions and coverage of TEs on the genome and transcriptome (lncRNAs and mRNAs) were then annotated with the custom library by using RepeatMasker (RepeatMasker -open-4.0.5) with a cutoff value of 250 . The overlaps of TEs with protein coding $(21,087)$ and lncRNA $(14,477)$ gene introns and exons, and their flanking regions (5 kb upstream and $3 \mathrm{~kb}$ downstream), mRNAs (45,788), lncRNAs $(29,585)$, CDS and UTRs (21,087 protein coding genes) were determined by intersecting these sets with TE annotations (described above) by using bedtools. Only overlaps of minimum 25 bp were retained.

The protein coding genes and TE chimeric transcripts in the pig genome were identified according to a high standard annotation strategy. Generally, the intersection between the coordinates of ESTs and protein coding genes were calculated, and only the ESTs with $90 \%$ of their coordinates overlapping with those of protein coding genes were retained; the remaining ESTs were then aligned to the mRNAs of protein coding genes by using Blat (-oneOff $=1,-$ minMatch $=4,-$ minScore $=90,-$ minIdentity $=$ 95), and only the ESTs with more than 70\% coverage of alignments with mRNAs were retained. Finally, these ESTs 
and mRNAs of protein coding genes were annotated de novo by using RepeatMasker (-cutoff 250 , -nolow) with the custom library. The ESTs with over $80 \%$ of TE coverage, which may be completely transcribed from an active TE element, were also discarded. The remaining ESTs and mRNAs with at least 50 bases marked by repeats designated as TE chimeric transcripts were retained for statistical analysis. This strategy allows elimination of all the TE-cassettes that are inserted into protein coding genes but do not correspond to a protein sequence, or those that correspond to putative transcriptionally active TEs. Thus, overestimation of TE insertions in the protein coding genes of the pig was avoided.

\section{Age estimation}

The average divergence and insertion ages of retrotransposons were estimated based on the divergence from consensus sequences by using RepeatMasker, and corrected as reported previously [103]. The average number of substitutions per site (K) for each fragment was estimated according to the divergence levels reported by RepeatMasker using the one-parameter Jukes-Cantor formula $K=-300 / 4 \times \operatorname{Ln}(1-D \times 4 / 300)$, as described previously [29], where $D$ represents the proportion of sites that differ between the fragmented repeat and the consensus sequence. Rough estimates of the ages of retrotransposons were obtained by using the equation $t=K / 2 r$ [103], where $t$ is the age and $r$ is the average nucleotide substitution rate of mammalian genomes. Analysis of mammal genomes has shown that the rate of single nucleotide substitution remains relatively constant $\left(1-2.2 \times 10^{-9}\right.$ substitutions/site/year) [104, 105]. In the present study, we assumed an average mutation rate of $2.2 \times 10^{-9}$ per site per year for pigs. These time estimations do not necessarily represent exact dates, but provide relative approximations and simple calculations.

\section{Insertion polymorphism detection of Young retrotransposons}

Seven domestic pig breeds (including Yorkshire, Landrace, Meishan, Shawutou, Jiangquhai, Sujiang, and Bama) and two wild boars were used for insertion polymorphism detection of the three youngest retrotransposon families/subfamilies (L1D1, SINEA1, and ERV6B) by PCR. Each domestic breed had three individuals. Meishan, Shawutou, and Jiangquhai pigs are native Chinese pig breeds from Jiangsu Province; the Sujiang pig is a newly established breed based on Duroc and Jiangquhai bloodlines; Bama pigs are miniature pigs from Guangxi Province; the wild boar was from Anhui Province; and the Landrace and Yorkshire pigs were from a breeding farm in Anhui Province. DNA was isolated from ear or blood samples of each sample by using the MiniBEST Universal Genomic DNA Extraction Kit
Ver.5.0 (TaKaRa, Dalian, China). The concentration and quality of the DNA were measured using a spectrophotometer and electrophoresis in agarose gel. The primers (listed in Additional file 2: Table S5) designed for detection are shown in Additional file 2: Figure S5. For L1D1 and ERV6B, we designed a primer in its flanking region and another in their 5'UTR/LTR. For SINEA1, the primers were designed in its flanking regions, which span the SINEA1 insertion.

\section{Plasmid construction \\ Luciferase reporter vectors}

Eight sense 5'UTRs of L1D family (one each from L1D1, L1D4, L1D6, and L1D7, and two from L1D2 and L1D3, respectively) and four antisense $5^{\prime}$ UTRs of L1D family (L1D1, L1D2, L1D3, and L1D7), sense and antisense LTRs from both ERV6A and ERV6 were cloned from pig genomic DNA by nested PCR with Phanta Max Super-Fidelity DNA Polymerase (Vazyme, Nanjing, China). Two rounds of specific primers were designed according to the sequences from their genomic coordinates, and the restriction enzymes MluI/KpnI or MluI/ SmaI were added to the $5^{\prime}$-flank of the second round of primers. We also cloned two 5'UTRs from human L1 (L1.3 and L1-M) [106] and one 5'UTR from mouse (mL1) [62] for positive control. Primers and their genomic coordinates are listed in Additional file 2: Table S6. PCR products were cloned into the cloning vector $\mathrm{pLB}$ (VT205; Tiangen, Beijing, China), and the correctness of the sequences was confirmed by sequencing. The 5'UTR or LTR was excised from the pLB vector by restriction enzyme digestion and inserted upstream of the firefly luciferase coding sequence in the pGL3-enhancer vector (Promega, Madison, WI, USA), respectively. The recombinant vectors were confirmed by sequencing again. The schematics of the vectors are shown in Fig. 4a.

\section{Retrotransposon activity verification vectors}

A total of five vectors (pL1, pL1CMV, phL1, hL1, and mhL1) were used in the retrotransposon activity analysis. The hL1 (99-PUR-RPS-pBlaster1) and mhL1 (99-PURJM111-5-15, the same as hL1, but ORF1 mutant and has no retrotransposon activity) were gifts from John $\mathrm{L}$. Goodier and Haig H. Kazazian, Jr. [50] and were used as positive and negative controls, respectively. The 5'UTR and 3'UTR of pig L1D1, and the middle region of L1D1, including ORF1, IGR, and ORF2, were amplified by PCR from pig genomic coordinate (Sscrofa11.1 chr9:9523 5839-95,244,641), respectively. Human IGR was cloned from hL1 (99-PUR-RPS-pBlaster1). The human IGR was inserted into the middle of ORF1 and ORF2 of pig L1 by overlap PCR. The CMV promoter was cloned from the pEGFP-N1. These fragments were inserted into TA cloning vectors and confirmed by sequencing. They were 
assembled into vectors of pL1, pL1-CMV, and phL1 by ligase with the designed restriction enzyme sites. The primers used to amplify the fragments described above are listed in Additional file 2: Table S7. The pL1 vector contains 5'UTR, ORF1, IGR, ORF2 and 3'UTR of L1, which are all cloned from the pig genome (L1D1). The pL1-CMV is the same as pL1, but the $5^{\prime}$ UTR of pig L1 was replaced with the CMV promoter. The vector phL1 is a chimeric vector derived by the CMV promoter, the two ORFs and 3'UTR were from pig L1, and the IGR was from human L1 (99-PUR-RPS-pBlaster1). All vectors contained two selective cassettes (mBlast and Puro) for two-round selections. The schematics of these vectors are listed in Fig. 2a.

\section{Cell culture}

HeLa cells (CCL-2; ATCC, USA) and MEF cells (kindly provided by Dr. Han Wu from Chinese Academy of Medical Sciences) were cultured in DMEM medium supplemented with $10 \%$ fetal bovine serum (FBS), $100 \mathrm{U} / \mathrm{mL}$ penicillin and $0.1 \mathrm{mg} / \mathrm{mL}$ streptomycin. PEF cells (kindly provided by Dr. Kui Li from Chinese Academy of Agricultural Sciences) were grown in DMEM containing 20\% FBS, $1 \mathrm{x}$ nonessential amino acids, $1 \mathrm{mM}$ sodium pyruvate, and 2 mM L-glutamine. PK15 cells (kindly provided by Dr. Han Wu from Chinese Academy of Medical Sciences) were grown in DMEM containing 10\% FBS and $2 \mathrm{mM}$ L-glutamine, $100 \mathrm{U} / \mathrm{mL}$ penicillin, and $0.1 \mathrm{mg} / \mathrm{mL}$ streptomycin. Culture of cells was maintained in a humidified atmosphere with $5 \% \mathrm{CO}_{2}$ in air at $37^{\circ} \mathrm{C}$. All cell culture reagents used were purchased from Thermo Fisher Scientific (Waltham, MA, USA).

\section{Retrotransposition assay}

Retrotransposition assays were performed as described by [50]. Briefly, $3 \times 10^{5}$ HeLa cells or PK15 cells were seeded onto each well of 6-well plates 1 day prior to transfection, and transfected with $3 \mu \mathrm{g}$ of DNA (plasmid pL1/ pL1-CMV/phL1/hL1/mhL1) using the FuGene HD transfection reagent (Promega) (cell confluence $>80 \%$ on day of transfection). Then, $48 \mathrm{~h}$ after transfection, transfected cells were replated onto T75 flasks and selected in $3 \mu \mathrm{g} / \mathrm{mL}$ puromycin (InvivoGen, San Diego, CA, USA) for HeLa and $4 \mu \mathrm{g} / \mathrm{mL}$ puromycin for PK15 cells. After 5 days of selection, both the HeLa and PK15 cells were selected again in $4 \mu \mathrm{g} / \mathrm{mL}$ blasticidin (InvivoGen) for 10 days. The blasticidin-resistant colonies were then stained with $0.4 \%$ Giemsa (Solarbio, Beijing, China) and counted. For the transposition activity assay, at least three independent experiments were performed, and three independent parallel groups were set up for each experiment.

\section{Promoter activity assay}

The promoters activity of 5'UTR from young pig L1 subfamilies and LTRs from ERV6 were tested using the Dual-Luciferase Reporter Assay System. In short, $3 \times 10^{5} \mathrm{HeLa}, \mathrm{MEF}, \mathrm{PEF}$, or PK15 cells were seeded onto each well of 6 -well plates 1 day prior to transfection and then transfected with $2 \mu \mathrm{g}$ of plasmid fire luciferase (pGL3-LTR/5'UTR-Luc/pGL3-control/pGL3enhancer) and Renilla luciferase (pRL-TK) at a 10:1 ratio using the FuGene HD transfection reagent. After $48 \mathrm{~h}$ post-transfection, the cells were lysed and harvested. The luciferase activity from the lysed cells was detected according to the protocol of the Dual-Luciferase $^{\bullet}$ Reporter Assay System kit (Promega) with a Modulus $^{\text {Th }}$ II Microplate Multimode Reader (Turner Biosystems, Sunnyvale, CA, USA). More than three independent experiments were performed.

\section{Real-time quantitative PCR}

To evaluate the sense and antisense expression profiles of young retrotransposon, including L1D, SINEA, and ERV6, the primers were designed according to the conserved regions of 5 'UTR, ORF1, and ORF2 of L1D, SINEA, and LTR, gag, pol, and env of ERV6. Expression levels were measured by real-time qPCR. Primer design for RT and $\mathrm{QPCR}$ detection are shown in Fig. 5a. Primer sequences and their genomic coordinates are listed in Additional file 2: Table S8. GAPDH was used as an internal control. Total RNAs were isolated from the multiple tissues of three female and three male pigs (Bama, Guangxi Province, China) at 3 months of age, and PK15 and PEF cells by using standard Trizol methods (Invitrogen, Carlsbad, CA, USA). To synthesize the first strand of cDNA, $1 \mu \mathrm{g}$ of total RNA was reverse-transcribed by using genespecific primers with the FastQuant RT Kit (with gDNase) (TianGen). The RNA treated with DNase and without RT was used as template of PCR to confirm no DNA contamination. The real-time qPCR was then performed using SYBR Premix Ex Taq II (Tli RNaseH Plus) (TaKaRa) with an Applied Biosystems $^{\circ} 7500$ Real-Time PCR System (Applied Biosystems, Foster City, CA, USA).

\section{Statistical analyses}

One-way ANOVA was used to determine differences in clones between groups in the retrotransposition assay using SPSS (version 16.0; Chicago, IL, USA). The LSD method was used for post-test analysis. The frequency difference of sense and antisense TE insertions was compared by using the $\chi^{2}$ test. A $p$ value $<0.05$ was considered to be significant in all analyses. 


\section{Additional files}

Additional file 1: The consensus sequences or representative sequences of L1, SINE and ERV. (DOCX $200 \mathrm{~kb}$ )

Additional file 2: Table S1. Detail information of pig $L 1$ families in the pig genome. Table S2. (SINE name and length information after reclassification). Table S3. (Detailed information on ERV in the pig genome). Table S4. (Composition of interspersed repeats in the pig genome). Table S5. (Primers for insertion polymorphism detection of youngest retrotransposons). Table S6. (Primers for promoter activity assay of LTR /5'UTR of ERV/ L1). Table S7. (Primers for retrotransposon assay of L1). Table S8. (Primers for detection of the expression of retrotransposons by RT-qPCR). Figure S1. (Comparison and reclassification of SINE transposons derived from tRNA in Repbase libraries by sequence alignment). Figure S2. (ERVs were classified into three classes based on the NJ phylogenetic tree). Figure S3. (Schematic of the protein structures of full-length ERV6 members in pig genome). Figure S4. (Retrotransposon distribution in pig genome and the impact on genes). Figure S5. (Primers designed for the youngest retrotransposons insertion polymorphism detection). (DOCX $2580 \mathrm{~kb})$

\section{Abbreviations}

env: Envelope protein; ERVs: Endogenous retroviruses; gag: Group-specific antigen; IGR: Intergenic region; LINEs: Long interspersed nuclear elements; LTRs: Long terminal repeats; MIR: Mammalian-wide interspersed repeat; Mya: Million years ago; ORF1: Open reading frame 1; pol: Polymerase; RT: Reverse transcription; SINEs: Short interspersed nuclear elements; TEs: Transposable elements

\section{Acknowledgments}

We would like to thank Dr. Astrid Engel (Tulane University) for advice on retrotransposition assays and Dr. John L. Goodier and Haig H. Kazazian for the hL1 (99-PUR-RPS-pBlaster1) and mhL1 (99-PUR-JM111-5-15) vectors.

\section{Funding}

This work was supported by the Natural Science Foundation of China (31572364 31872977); and the Priority Academic Program Development of Jiangsu Higher Education Institutions.

\section{Authors' contributions}

CS conceived the study, KL, JM, KM and BG participated in its design, CC and WW performed the experiments and analyses. All authors read, gave comments and helped to revise the final version of the manuscript. All authors read and approved the final manuscript.

\section{Ethics approval and consent to participate}

Animal care and use was approved by the Animal Care and Use Committee of Yangzhou University.

\section{Consent for publication}

Not applicable.

\section{Competing interests}

The authors declare that they have no competing interests.

\section{Publisher's Note}

Springer Nature remains neutral with regard to jurisdictional claims in published maps and institutional affiliations.

\section{Author details}

${ }^{1}$ Institute of Animal Mobilome and Genome, College of Animal Science \& Technology, Yangzhou University, Yangzhou 225009, Jiangsu, China. ${ }^{2}$ Leibniz Institute for Farm Animal Biology (FBN), 18196 Dummerstorf, Germany. ${ }^{3}$ Life Science Center, University of Missouri, Columbia, MO 65211, USA. ${ }^{4}$ Institute of Animal Science, Chinese Academy of Agricultural Sciences, Beijing, China.
Received: 14 February 2019 Accepted: 11 April 2019

Published online: 06 May 2019

\section{References}

1. Kazazian HH. Mobile Elements: Drivers of Genome Evolution. Science. 2004; 80:1626-32.

2. Wicker T, Sabot F, Hua-Van A, Bennetzen JL, Capy P, Chalhoub B, et al. A unified classification system for eukaryotic transposable elements. Nat Rev Genet. 2007:8:973-82

3. Michael TP. Plant genome size variation: bloating and purging DNA. Brief Funct Genomic Proteomic. 2014;13:308-17.

4. Gao B, Shen D, Xue S, Chen C, Cui H, Song C. The contribution of transposable elements to size variations between four teleost genomes. Mob DNA. 2016;7:4.

5. Chalopin D, Naville M, Plard F, Galiana D, Volff JN. Comparative analysis of transposable elements highlights mobilome diversity and evolution in vertebrates. Genome Biol Evol. 2015;7:567-80.

6. Lander ES, Linton LM, Birren B, Nusbaum C, Zody MC, Baldwin J, et al. Initial sequencing and analysis of the human genome. Nature. 2001;409(6822): 860-921.

7. Schnable PS, Ware D, Fulton RS, Stein JC, Wei F, Pasternak S, et al. The B73 maize genome: Complexity, diversity, and dynamics. Science. 2009:326:1112-5.

8. Platt RN, Vandewege MW, Ray DA. Mammalian transposable elements and their impacts on genome evolution. Chromosome Res. 2018:26:25-43.

9. Bennetzen $J \mathrm{~L}$, Wang $\mathrm{H}$. The contributions of transposable elements to the structure, function, and evolution of plant genomes. Annu Rev Plant Biol. 2014:65:505-30.

10. Majumdar S, Singh A, Rio DC. The human THAP9 gene encodes an active Pelement DNA transposase. Science. 2013:339:446-8.

11. Henssen AG, Henaff $E$, Jiang E, Eisenberg AR, Carson JR, Villasante $C M$, et al. Genomic DNA transposition induced by human PGBD5. Elife. 2015:4:e10565.

12. Alzohairy AM, Gyulai G, Jansen RK, Bahieldin A. Transposable elements domesticated and neofunctionalized by eukaryotic genomes. Plasmid. 2013;69:1-15.

13. Pathak D, Ali S. Rsal repetitive DNA in Buffalo Bubalus bubalis representing retrotransposons, conserved in bovids, are part of the functional genes. BMC Genomics. 2011;12:338.

14. Kim DS, Kim TH, Huh JW, Kim IC, Kim SW, Park HS, et al. LINE FUSION GENES: a database of LINE expression in human genes. BMC Genomics. 2006;7:139.

15. Bourque G, Burns KH, Gehring M, Gorbunova V, Seluanov A, Hammell M, et al. Ten things you should know about transposable elements. Genome Biol. 2018;19:199.

16. Cowley M, Oakey RJ. Transposable Elements Re-Wire and Fine-Tune the Transcriptome. PLoS Genet. 2013:9:e1003234.

17. Göke J, Ng HH. CTRL+INSERT: retrotransposons and their contribution to regulation and innovation of the transcriptome. EMBO Rep. 2016:17:1131-44.

18. Sundaram V, Cheng Y, Ma Z, Li D, Xing X, Edge P, et al. Widespread contribution of transposable elements to the innovation of gene regulatory networks. Genome Res. 2014;24:1963-76.

19. Jacques PÉ, Jeyakani J, Bourque G. The majority of primate-specific regulatory sequences are derived from transposable elements. PLoS Genet. 2013;9:e1003504.

20. Byun HM, Heo K, Mitchell KJ, Yang AS. Mono-allelic retrotransposon insertion addresses epigenetic transcriptional repression in human genome. J Biomed Sci. 2012:19:13.

21. Fukuda K, Inoguchi Y, Ichiyanagi K, Ichiyanagi T, Go Y, Nagano M, et al. Evolution of the sperm methylome of primates is associated with retrotransposon insertions and genome instability. Hum Mol Genet. 2017;26:3508-19.

22. Inamura K, Yamauchi M, Nishihara R, Lochhead P, Qian ZR, Kuchiba A, et al. Tumor LINE-1 methylation level and microsatellite instability in relation to colorectal cancer prognosis. J Natl Cancer Inst. 2014;106(9).

23. Hur K, Cejas P, Feliu J, Moreno-Rubio J, Burgos E, Boland CR, et al. Hypomethylation of long interspersed nuclear element-1 (LINE-1) leads to activation of protooncogenes in human colorectal cancer metastasis. Gut. 2014;63:635-46

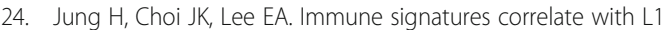
retrotransposition in gastrointestinal cancers. Genome Res. 2018;28:1136-46.

25. Hadjiargyrou M, Delihas $N$. The intertwining of transposable elements and non-coding RNAs. Int J Mol Sci. 2013;14:13307-28.

26. Kelley D, Rinn J. Transposable elements reveal a stem cell-specific class of long noncoding RNAs. Genome Biol. 2012;13:R107. 
27. Kapusta A, Kronenberg Z, Lynch VJ, Zhuo X, Ramsay LA, Bourque G, et al. Transposable elements are major contributors to the origin, diversification, and regulation of vertebrate long noncoding RNAs. PLoS Genet. 2013;9: e1003470.

28. Kannan S, Chernikova D, Rogozin IB, Poliakov E, Managadze D, Koonin EV, et al. Transposable Element Insertions in Long Intergenic Non-Coding RNA Genes. Front Bioeng Biotechnol. 2015;3:71.

29. Waterston RH, Lindblad-Toh K, Birney E, Rogers J, Abril JF, Agarwal P, et al. Initial sequencing and comparative analysis of the mouse genome. Nature. 2002;420:520-62

30. Groenen MAM, Archibald AL, Uenishi H, Tuggle CK, Takeuchi Y, Rothschild MF, et al. Analyses of pig genomes provide insight into porcine demography and evolution. Nature. 2012;491:393-8.

31. Warren WC, Hillier LDW, Marshall Graves JA, Birney E, Ponting CP, Grützner $F$, et al. Genome analysis of the platypus reveals unique signatures of evolution. Nature. 2008;453:175-83.

32. Renfree MB, Papenfuss AT, Deakin JE, Lindsay J, Heider T, Belov K, et al. Genome sequence of an Australian kangaroo, Macropus eugenii, provides insight into the evolution of mammalian reproduction and development. Genome Biol. 2011;12:R81.

33. Adey NB, Schichman SA, Graham DK, Peterson SN, Edgell MH, Hutchison CA. r. Rodent $\mathrm{L} 1$ evolution has been driven by a single dominant lineage that has repeatedly acquired new transcriptional regulatory sequences. Mol Biol Evol. 1994;11:778-89.

34. Sookdeo A, Hepp CM, McClure MA, Boissinot S. Revisiting the evolution of mouse LINE-1 in the genomic era. Mob DNA. 2013:4:3.

35. Boissinot S, Sookdeo A. The evolution of LINE-1 in vertebrates. Genome Biol Evol. 2016:8:3485-507.

36. Richardson SR, Doucet AJ, Kopera HC, Moldovan JB, Garcia-Perez JL, Moran $J V$. The Influence of LINE-1 and SINE Retrotransposons on Mammalian Genomes. Microbiol Spectr. 2015;3:1165-1208.

37. Cantrell MA, Scott LA, Brown CJ, Martinez AR, Wichman HA. Loss of LINE-1 activity in the megabats. Genetics. 2008;178:393-404.

38. Gogolevsky KP, Vassetzky NS, Kramerov DA. 55 rRNA-derived and tRNA-derived SINEs in fruit bats. Genomics. 2009:93:494-500.

39. Ohshima K, Hattori M, Yada T, Gojobori T, Sakaki Y, Okada N. Wholegenome screening indicates a possible burst of formation of processed pseudogenes and Alu repeats by particular L1 subfamilies in ancestral primates. Genome Biol. 2003:4:R74.

40. Penzkofer $T$, Jäger M, Figlerowicz M, Badge R, Mundlos S, Robinson PN, et al. L1Base 2: more retrotransposition-active LINE-1s, more mammalian genomes. Nucleic Acids Res. 2017;45:D68-73.

41. Huang CRL, Burns KH, Boeke JD. Active transposition in genomes. Annu Rev Genet. 2012;46:651-75

42. Gentles AJ, Wakefield MJ, Kohany O, Gu W, Batzer MA, Pollock DD, et al. Evolutionary dynamics of transposable elements in the short-tailed opossum Monodelphis domestica. Genome Res. 2007:17:992-1004.

43. Yang L, Brunsfeld J, Scott LA, Wichman H. Reviving the dead: history and reactivation of an extinct L1. PLoS Genet. 2014;10:e1004395.

44. Stocking C, Kozak CA. Endogenous retroviruses: Murine endogenous retroviruses. Cell Mol Life Sci. 2008;65:3383-98.

45. Fang $X$, Mou Y, Huang Z, Li Y, Han L, Zhang Y, et al. The sequence and analysis of a Chinese pig genome. Gigascience. 2012;1:16.

46. Dewannieux M, Esnault C, Heidmann T. LINE-mediated retrotransposition of marked Alu sequences. Nat Genet. 2003;35:41-8.

47. Dewannieux M, Heidmann T, Yaniv M. L1-mediated retrotransposition of murine B1 and B2 SINEs recapitulated in cultured cells. J Mol Biol. 2005:349:241-7.

48. Vassetzky NS, Kramerov DA. CAN - a pan-carnivore SINE family. Mamm Genome. 2002;13:50-7.

49. Naas TP, Deberardinis RJ, Moran JV, Ostertag EM, Kingsmore SF, Seldin MF, et al. An actively retrotransposing, novel subfamily of mouse L1 elements. EMBO J. 1998:17:590-7.

50. Goodier JL, Zhang L, Vetter MR, Kazazian HH. LINE-1 ORF1 protein localizes in stress granules with other RNA-binding proteins, including components of RNA interference RNA-induced silencing complex. Mol Cell Biol. 2007;27:6469-83.

51. Heras SR, Maclas S, Plass M, Fernandez N, Cano D, Eyras E, et al. The microprocessor controls the activity of mammalian retrotransposons. Nat Struct Mol Biol. 2013:20:1173-83.

52. Takeuchi $Y$, Patience $C$, Magre $S$, Weiss R, Banerjee $P$, LeTissier $P$, et al. Host range and interference studies of three classes of pig endogenous retrovirus. J Acquir Immune Defic Syndr Hum Retrovirology. 1999;20:A8.
53. Khan H, Smit A, Boissinot S. Molecular evolution and tempo of amplification of human LINE-1 retrotransposons since the origin of primates. Genome Res. 2006;16:78-87.

54. Katzourakis A, Gifford RJ, Tristem M, Thomas M, Gilbert P, Pybus OG Macroevolution of complex retroviruses. Science. 2009;325:1512

55. Tarlinton RE, Meers J, Young PR. Retroviral invasion of the koala genome. Nature. 2006;442:79-81.

56. Palmarini M, Sharp JM, De Las HM, Fan $H$. Jaagsiekte sheep retrovirus is necessary and sufficient to induce a contagious lung cancer in sheep. J Virol. 1999;73(8):6964-72.

57. Anai Y, Ochi H, Watanabe S, Nakagawa S, Kawamura M, Gojobori T, et al. Infectious endogenous retroviruses in cats and emergence of recombinant viruses. J Virol. 2012:86:8634-44.

58. Niu D, Wei HJ, Lin L, George $H$, Wang $T$, Lee $I H$, et al. Inactivation of porcine endogenous retrovirus in pigs using CRISPR-Cas9. Science. 2017;357:1303-7.

59. Lopata K, Wojdas E, Nowak R, Lopata P, Mazurek U. Porcine endogenous retrovirus (PERV)-molecular structure and replication strategy in the context of retroviral infection risk of human cells. Front Microbiol. 2018;9:730.

60. Gerdes P, Richardson SR, Mager DL, Faulkner GJ. Transposable elements in the mammalian embryo: pioneers surviving through stealth and service. Genome Biol. 2016;17:100

61. Criscione SW, Theodosakis N, Micevic G, Cornish TC, Burns KH, Neretti N, et al. Genome-wide characterization of human L1 antisense promoter-driven transcripts. BMC Genomics. 2016;17:463.

62. Li J, Kannan M, Trivett AL, Liao H, Wu X, Akagi K, et al. An antisense promoter in mouse $\mathrm{L} 1$ retrotransposon open reading frame-1 initiates expression of diverse fusion transcripts and limits retrotransposition. Nucleic Acids Res. 2014; 42:4546-62.

63. Speek M. Antisense promoter of human L1 retrotransposon drives transcription of adjacent cellular genes. Mol Cell Biol. 2001;21:1973-85.

64. Ha HS, Huh JW, Kim DS, Kang DW, Cho BW, Kim HS. Promoter activity of the long terminal repeats of porcine endogenous retroviruses of the Korean domestic pig. Mol Cells. 2007;24:148-51.

65. Jung YD, Ha HS, Park SJ, Oh KB, Im GS, Kim TH, et al. Identification and promoter analysis of PERV LTR subtypes in NIH-miniature pig. Mol Cells. 2013;35:99-105.

66. Feschotte $\mathrm{C}$. Transposable elements and the evolution of regulatory networks. Nat Rev Genet. 2008;9(5):397-405.

67. Hirsch CD, Springer NM. Transposable element influences on gene expression in plants. Biochim Biophys Acta Gene Regul Mech. 1860;2017:157-65.

68. Thompson PJ, Macfarlan TS, Lorincz MC. Long Terminal Repeats: From Parasitic Elements to Building Blocks of the Transcriptional Regulatory Repertoire. Mol Cell. 2016;62(5):766-76

69. Wu Q, Smith NA, Zhang D, Zhou C, Wang M-B. Root-Specific Expression of a Jacalin Lectin Family Protein Gene Requires a Transposable Element Sequence in the Promoter. Genes (Basel). MDPI. 2018;9:550

70. Li ZW, Hou XH, Chen JF, Xu YC, Wu Q, Gonzalez J, et al. Transposable elements contribute to the adaptation of arabidopsis thaliana. Genome Biol Evol. 2018:10:2140-50

71. Bao J, Yan W. Male germline control of transposable Elements1. Biol Reprod. 2012:86(162):1-14

72. Zakrzewski F, Schmidt M, Van Lijsebettens M, Schmidt T. DNA methylation of retrotransposons, DNA transposons and genes in sugar beet (Beta vulgaris L.) Plant J. 2017;90:1156-75

73. Yang N, Kazazian HH. L1 retrotransposition is suppressed by endogenously encoded small interfering RNAs in human cultured cells. Nat Struct Mol Biol. 2006;13:763-71.

74. Sironen A, Vilkki J, Bendixen C, Thomsen B. Infertile Finnish Yorkshire boars carry a full-length LINE-1 retrotransposon within the KPL2 gene. Mol Gen Genomics. 2007:278(4):385-91.

75. Koepfli K-P, Paten B, O'Brien SJ. The genome 10K project: a way forward. Annu Rev Anim Biosci. 2015:3:57-111.

76. Ngamphiw C, Tongsima S, Mutirangura A. Roles of intragenic and intergenic L1s in mouse and human. PLoS One. 2014:9:e113434.

77. Hancks DC, Kazazian HH. Roles for retrotransposon insertions in human disease. Mob DNA. 2016;7:9

78. Gray MM, Sutter NB, Ostrander EA, Wayne RK. The IGF1 small dog haplotype is derived from middle eastern grey wolves. BMC Biol. 2010;8:16.

79. Clark LA, Wahl JM, Rees CA, Murphy KE. From the cover: retrotransposon insertion in SILV is responsible for merle patterning of the domestic dog. Proc Natl Acad Sci. 2006;103:1376-81. 
80. Murphy SC, Evans JM, Tsai KL, Clark LA. Length variations within the merle retrotransposon of canine PMEL: correlating genotype with phenotype. Mob DNA. 2018:9:26

81. Wang Z, Qu L, Yao J, Yang X, Li G, Zhang Y, et al. An EAV-HP insertion in 5' flanking region of SLCO1B3 causes blue eggshell in the chicken. PLoS Genet. 2013;9:e1003183.

82. Giuffra E, Törnsten A, Marklund S, Bongcam-Rudloff E, Chardon P, Kijas JMH, et al. A large duplication associated with dominant white color in pigs originated by homologous recombination between LINE elements flanking KIT. Mamm Genome. 2002;13:569-77.

83. Sironen A, Uimari P, Iso-Touru T, Vilkki J. L1 insertion within SPEF2 gene is associated with increased litter size in the Finnish Yorkshire population. J Anim Breed Genet. 2012;129:92-7.

84. Almeida LM, Silva IT, Silva WA, Castro JP, Riggs PK, Carareto CM, et al. The contribution of transposable elements to Bos taurus gene structure. Gene. 2007:390:180-9.

85. Burns KH, Boeke JD. Human transposon tectonics. Cell. 2012:149:740-52

86. Levy A, Sela N, Ast G. TranspoGene and microTranspoGene: Transposed elements influence on the transcriptome of seven vertebrates and invertebrates. Nucleic Acids Res. 2008;36:D47-D52.

87. Medstrand P, Van De Lagemaat LN, Mager DL. Retroelement distributions in the human genome: variations associated with age and proximity to genes. Genome Res. 2002;12:1483-95

88. Ganesh S, Svoboda P. Retrotransposon-associated long non-coding RNAs in mice and men. Pflugers Arch Eur J Physiol. 2016;468:1049-60.

89. He S, Gu W, Li Y, Zhu H. ANRIL/CDKN2B-AS shows two-stage clade-specific evolution and becomes conserved after transposon insertions in simians. BMC Evol Biol. 2013;13:247.

90. Zucchelli S, Cotella D, Takahashi H, Carrieri C, Cimatti L, Fasolo F, et al. SINEUPS: a new class of natural and synthetic antisense long non-coding RNAs that activate translation. RNA Biol. 2015;12:771-9.

91. Podbevšek P, Fasolo F, Bon C, Cimatti L, Reißer S, Carninci P, et al. Structural determinants of the SINE B2 element embedded in the long non-coding RNA activator of translation AS Uchl1. Sci Rep. 2018;8:3189.

92. Kim EZ, Wespiser AR, Caffrey DR. The domain structure and distribution of Alu elements in long noncoding RNAs and mRNAs. RNA. 2016;22:254-64.

93. Faulkner GJ, Kimura Y, Daub CO, Wani S, Plessy C, Irvine KM, et al. The regulated retrotransposon transcriptome of mammalian cells. Nat Genet. 2009:41:563-71.

94. Lavi E, Carmel L. Alu exaptation enriches the human transcriptome by introducing new gene ends. RNA Biol. 2018;15:715-25.

95. Smalheiser NR, Torvik VI. Mammalian microRNAs derived from genomic repeats. Trends Genet. 2005;21:322-6.

96. Smalheiser NR, Torvik VI. Alu elements within human mRNAs are probable microRNA targets. Trends Genet. 2006;22:532-6.

97. Rho M, Tang H. MGEScan-non-LTR: computational identification and classification of autonomous non-LTR retrotransposons in eukaryotic genomes. Nucleic Acids Res. 2009;37:e143.

98. Kent WJ. BLAT - the BLAST-like alignment tool. Genome Res. 2002;12:656-64.

99. Ellinghaus D, Kurtz S, Willhoeft U. LTRharvest, an efficient and flexible software for de novo detection of LTR retrotransposons. BMC Bioinf. 2008;9:18.

100. Sperber GO, Airola T, Jern P, Blomberg J. Automated recognition of retroviral sequences in genomic data - RetroTector@. Nucleic Acids Res. 2007;35:4964-76.

101. Larkin MA, Blackshields G, Brown NP, Chenna R, Mcgettigan PA, McWilliam $H$, et al. Clustal W and Clustal X version 2.0. Bioinformatics. 2007;23:2947-8.

102. Kumar S, Stecher G, Tamura K. MEGA7: molecular evolutionary genetics analysis version 7.0 for bigger datasets. Mol Biol Evol. 2016;33:1870-4.

103. Kimura M. A simple method for estimating evolutionary rates of base substitutions through comparative studies of nucleotide sequences. J Mol Evol. 1980:16:111-20.

104. Kumar S, Subramanian S. Mutation rates in mammalian genomes. Proc Natl Acad Sci. 2002;99:803-8.

105. Liu G, Thomas J, Touchman J, Blakesley B, Bouffard G, Beckstrom-Sternberg $S$, et al. Analysis of primate genomic variation reveals a repeat-driven expansion of the human genome. Genome Res. 2003;13:358-68.

106. Lavie L, Maldener E, Brouha B, Meese EU, Mayer J. The human L1 promoter: variable transcription initiation sites and a major impact of upstream flanking sequence on promoter activity. Genome Res. 2004;14:2253-60.

Ready to submit your research? Choose BMC and benefit from:

- fast, convenient online submission

- thorough peer review by experienced researchers in your field

- rapid publication on acceptance

- support for research data, including large and complex data types

- gold Open Access which fosters wider collaboration and increased citations

- maximum visibility for your research: over $100 \mathrm{M}$ website views per year

At BMC, research is always in progress.

Learn more biomedcentral.com/submissions 